\title{
PRECOVERS AND PREENVELOPES UNDER CHANGE OF RINGS
}

\author{
LIXIN MAO
}

\begin{abstract}
Let $R \rightarrow S$ be a ring homomorphism. We analyze the relationship between the precovers (preenvelopes) in the category of right $R$-modules and the counterparts in the category of right $S$-modules. Some applications are also given.
\end{abstract}

1. Introduction. (Pre)covers and (pre)envelopes were introduced in the early 1980s by Enochs [4] and, independently, by Auslander and Smalø [2]. Let $\mathcal{C}$ be a class of right $R$-modules and $M$ a right $R$-module. A homomorphism $\phi: C \rightarrow M$ is called a $\mathcal{C}$-precover of $M$ if $C \in \mathcal{C}$ and the abelian group homomorphism $\phi_{*}: \operatorname{Hom}_{R}\left(C^{\prime}, C\right) \rightarrow \operatorname{Hom}_{R}\left(C^{\prime}, M\right)$ is surjective for each $C^{\prime} \in \mathcal{C}$. A $\mathcal{C}$-precover $\phi: C \rightarrow M$ is said to be a $\mathcal{C}$-cover if every endomorphism $g: C \rightarrow C$ such that $\phi g=\phi$ is an isomorphism. Dually, we have the notions of a $\mathcal{C}$-preenvelope and a $\mathcal{C}$-envelope. In the representation theory of artin algebras, the usual terminology for a precover (respectively, a preenvelope) is a right (respectively, a left) approximation. Accordingly, the usual terminology for a cover (respectively, an envelope) is a minimal right (respectively, a minimal left) approximation. $\mathcal{C}$-covers and $\mathcal{C}$-envelopes may not exist in general, but, if they exist, they are unique up to isomorphism. (Pre)covers and (pre)envelopes provide a common framework for a number of classical notions such as projective covers and injective envelopes and turn out to be extremely fruitful for general module theory as well as for representation theory (see, e.g., $[\mathbf{2}, \mathbf{4}, \mathbf{5}, \mathbf{6}, \mathbf{1 3}]$ ).

2010 AMS Mathematics subject classification. Primary 16D90, 16E30, 18 G25.

Keywords and phrases. (Pre)cover, (pre)envelope, ring homomorphism, homomorphism of modules, tensor product of modules.

This research was supported by NSFC (Nos. 11171149 and 11371187), NSF of Jiangsu Province of China (No. BK2011068), Jiangsu 333 Project and Jiangsu Six Major Talents Peak Project.

Received by the editors on April 28, 2013, and in revised form on August 12, 2013. 
Let $R \rightarrow S$ be a ring homomorphism. Then $S$ becomes a canonical $R$-bimodule. Thus any right (respectively, left) $S$-module can be regarded as a right (respectively, left) $R$-module, and so a homomorphism of right (respectively, left) $S$-modules can also be regarded as a homomorphism of right (respectively, left) $R$-modules. Many results on the relationship between the (pre)covers ((pre)envelopes) in the category of $R$-modules and the counterparts in the category of $S$-modules have been obtained. For example, Würfel gave a characterization of those rings $R$ such that, for any ring homomorphism $R \rightarrow S$, the functor $\operatorname{Hom}_{R}(S,-)$ preserves injective envelopes [12]. Dempsey, Oyonarte and Song furthermore studied those rings $R$ such that for any ring homomorphism $R \rightarrow S$, the functor $\operatorname{Hom}_{R}(S,-)$ preserves injective envelopes or injective covers [3]. Zhou investigated relative preenvelopes under almost excellent extensions of rings [15]. In the present paper, we will consider more general settings, i.e., we will study the properties of relative (pre)covers and (pre)envelopes under change of rings using the functors $\operatorname{Hom}_{R}(S,-), \operatorname{Hom}_{R}(-, S)$ and $-\otimes_{R} S$.

Throughout this paper, all rings are associative with identity and all modules are unitary. $M_{R}$ (respectively, ${ }_{R} M$ ) denotes a right (respectively, left) $R$-module. $R \rightarrow S$ always means a ring homomorphism. For a right $R$-module $M$, we write $M^{*}=\operatorname{Hom}_{R}(M, S)$, $M^{* *}=\operatorname{Hom}_{S}\left(\operatorname{Hom}_{R}(M, S), S\right)$. There exists a canonical evaluation map $\delta_{M}: M \rightarrow M^{* *}$ defined by $\delta_{M}(x)(f)=f(x)$ for $x \in M$ and $f \in M^{*}$. $M$ is called $S$-reflexive if $\delta_{M}$ is an isomorphism. All classes of modules are assumed to be closed under isomorphisms. For a class $\mathcal{C}_{R}$ of right $R$-modules, we write $\operatorname{Hom}_{R}\left(S, \mathcal{C}_{R}\right)=\left\{\operatorname{Hom}_{R}(S, L): L \in \mathcal{C}_{R}\right\}$, $\mathcal{C}_{R} \otimes_{R} S=\left\{L \otimes_{R} S: L \in \mathcal{C}_{R}\right\},\left(\mathcal{C}_{R}\right)^{*}=\left\{M^{*}: M \in \mathcal{C}_{R}\right\}$ and $\left(\mathcal{C}_{R}\right)^{* *}=\left\{M^{* *}: M \in \mathcal{C}_{R}\right\}$.

Let $R \rightarrow S$ be a ring homomorphism, $M_{R}$ a right $R$-module and $N_{S}$ a right $S$-module.

There are a natural $R$-homomorphism $\varepsilon_{M}: \operatorname{Hom}_{R}(S, M) \rightarrow M_{R}$ defined by $\varepsilon_{M}(f)=f(1)$ for $f \in \operatorname{Hom}_{R}(S, M)$ and a natural $S$ homomorphism $\eta_{N}: N_{S} \rightarrow \operatorname{Hom}_{R}(S, N)$ defined by $\eta_{N}(y)(t)=y t$ for $y \in N$ and $t \in S$. It is not hard to verify that the composition of $R$-homomorphisms $N_{S} \stackrel{\eta_{N}}{\rightarrow} \operatorname{Hom}_{R}(S, N) \stackrel{\varepsilon_{N}}{\rightarrow} N_{R}$ is an identity and the composition of $S$-homomorphisms $\operatorname{Hom}_{R}(S, M) \stackrel{\eta_{\mathrm{Hom}_{R}(S, M)}^{\longrightarrow}}{\longrightarrow}$ $\operatorname{Hom}_{R}\left(S, \operatorname{Hom}_{R}(S, M)\right) \stackrel{\left(\varepsilon_{M}\right)_{*}}{\rightarrow} \operatorname{Hom}_{R}(S, M)$ is also an identity. 
On the other hand, there are a natural $S$-homomorphism $\mu_{N}: N \otimes_{R}$ $S \rightarrow N_{S}$ defined by $\mu_{N}(x \otimes t)=x t$ for $x \in N$ and $t \in S$ and a natural $R$-homomorphism $\nu_{M}: M_{R} \rightarrow M \otimes_{R} S$ defined by $\nu_{M}(y)=y \otimes 1$ for $y \in M$. It is easy to check that the composition of $R$-homomorphisms $N_{R} \stackrel{\nu_{N}}{\rightarrow} N \otimes_{R} S \stackrel{\mu_{N}}{\rightarrow} N_{S}$ is an identity and the composition of $S$ homomorphisms $M \otimes_{R} S \stackrel{\nu_{M} \otimes_{R} 1}{\rightarrow}\left(M \otimes_{R} S\right) \otimes_{R} S \stackrel{\mu_{M} \otimes_{R} S}{\rightarrow} M \otimes_{R} S$ is also an identity.

For unexplained concepts and notations, we refer the reader to $[1,5,6,7,10,13]$.

Let us describe the contents of the article in more detail.

In Section 2, we investigate the (pre)covers and (pre)envelopes under the covariant functor $\operatorname{Hom}_{R}(S,-)$ and the contravariant functor $\operatorname{Hom}_{R}(-, S)$. For example, let $R \rightarrow S$ be a ring homomorphism, $\mathcal{C}_{R}$ a class of right $R$-modules, $\mathcal{D}_{S}$ a class of right $S$-modules with $\left(\mathcal{D}_{S}\right)_{R} \subseteq \mathcal{C}_{R}$ and $\left.\operatorname{Hom}_{R}\left(S, \mathcal{C}_{R}\right)\right)_{S} \subseteq \mathcal{D}_{S}$. We prove that:

(i) Assume that $\varphi: M_{R} \rightarrow N_{R}$ is a right $R$-homomorphism with $M_{R} \in \mathcal{C}_{R}$. Then $\varphi_{*}: \operatorname{Hom}_{R}(S, M) \rightarrow \operatorname{Hom}_{R}(S, N)$ is a $\left(\operatorname{Hom}_{R}\left(S, \mathcal{C}_{R}\right)\right)_{S}$-precover if and only if $\varphi \varepsilon_{M}: \operatorname{Hom}_{R}(S, M) \rightarrow$ $N_{R}$ is a $\left(\operatorname{Hom}_{R}\left(S, \mathcal{C}_{R}\right)\right)_{R}$-precover.

(ii) Assume that $\varphi: M_{S} \rightarrow N_{S}$ is a right $S$-homomorphism with $N_{S} \in$ $\mathcal{D}_{S}$. Then $\varphi_{*} \eta_{M}: M_{S} \rightarrow \operatorname{Hom}_{R}(S, N)$ is a $\left(\operatorname{Hom}_{R}\left(S, \mathcal{C}_{R}\right)\right)_{S^{-}}$ preenvelope if and only if $\varphi: M_{R} \rightarrow N_{R}$ is a $\mathcal{C}_{R}$-preenvelope.

As a consequence, we obtain the behavior of cotorsion (injective) precovers and preenvelopes under change of rings.

In the case of the functor $\operatorname{Hom}_{R}(-, S)$, let $\mathcal{C}_{R}$ be a class of right $R$ modules, ${ }_{S} \mathcal{D}$ a class of left $S$-modules with $\left(\mathcal{C}_{R}\right)^{*} \subseteq{ }_{S} \mathcal{D}$ and $\left({ }_{S} \mathcal{D}\right)^{*} \subseteq$ $\mathcal{C}_{R}, \varphi: M_{R} \rightarrow N_{R}$ a right $R$-homomorphism with $N_{R} \in \mathcal{C}_{R}$. We obtain that $\varphi^{*}: N^{*} \rightarrow M^{*}$ is a $\left(\mathcal{C}_{R}\right)^{*}$-precover if and only if $\delta_{N} \varphi: M_{R} \rightarrow N^{* *}$ is a $\left(\mathcal{C}_{R}\right)^{* *}$-preenvelope.

Section 3 is devoted to the (pre)covers and (pre)envelopes under the covariant functor $-\otimes_{R} S$. For example, let $\varphi: R \rightarrow S$ be a ring homomorphism, $\mathcal{C}_{R}$ a class of right $R$-modules, $\mathcal{D}_{S}$ a class of right $S$-modules with $\left(\mathcal{D}_{S}\right)_{R} \subseteq \mathcal{C}_{R}$ and $\left(\mathcal{C}_{R} \otimes_{R} S\right)_{S} \subseteq \mathcal{D}_{S}$. We prove that:

(i) Suppose that $\varphi: M_{R} \rightarrow N_{R}$ is a right $R$-homomorphism with $N_{R} \in \mathcal{C}_{R}$. Then $\varphi \otimes_{R} 1: M \otimes_{R} S \rightarrow N \otimes_{R} S$ is a $\left(\mathcal{C}_{R} \otimes_{R} S\right)_{S^{-}}$ 
preenvelope if and only if $\nu_{N} \varphi: M_{R} \rightarrow N \otimes_{R} S$ is a $\left(\mathcal{C}_{R} \otimes_{R} S\right)_{R^{-}}$ preenvelope.

(ii) Suppose that $\varphi: M_{S} \rightarrow N_{S}$ is a right $S$-homomorphism with $M_{S} \in \mathcal{D}_{S}$. Then $\mu_{N}\left(\varphi \otimes_{R} 1\right): M \otimes_{R} S \rightarrow N_{S}$ is a $\left(\mathcal{C}_{R} \otimes_{R} S\right)_{S^{-}}$ precover if and only if $\varphi: M_{R} \rightarrow N_{R}$ is a $\mathcal{C}_{R}$-precover.

As a consequence, we get the properties of flat ( $F P$-injective) (pre)covers and (pre)envelopes under localization of rings.

In Section 4, we deal with the (pre)covers and (pre)envelopes under some kinds of special ring homomorphisms such as surjective ring homomorphisms and (almost) excellent extensions of rings. For example, let $R \rightarrow S$ be a surjective ring homomorphism, $\mathcal{C}_{R}$ a class of right $R$-modules, $\mathcal{D}_{S}$ a class of right $S$-modules with $\left(\mathcal{D}_{S}\right)_{R} \subseteq \mathcal{C}_{R}$ and $\varphi: M_{S} \rightarrow N_{S}$ a right $S$-homomorphism. We prove that:

(i) If $\left(\operatorname{Hom}_{R}\left(S, \mathcal{C}_{R}\right)\right)_{S} \subseteq \mathcal{D}_{S}$, then $\varphi: M_{S} \rightarrow N_{S}$ is a $\mathcal{D}_{S}$-preenvelope (respectively, $\mathcal{D}_{S}$-envelope) if and only if $\varphi: M_{R} \rightarrow N_{R}$ is a $\mathcal{C}_{R}$-preenvelope (respectively, $\mathcal{C}_{R}$-envelope).

(ii) If $\left(\mathcal{C}_{R} \otimes_{R} S\right)_{S} \subseteq \mathcal{D}_{S}$, then $\varphi: M_{S} \rightarrow N_{S}$ is a $\mathcal{D}_{S}$-precover

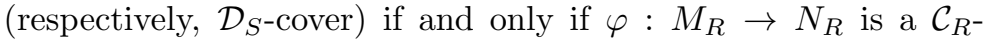
precover (respectively, $\mathcal{C}_{R^{-}}$cover).

On the other hand, let $S$ be an excellent extension of a subring $R, \mathcal{C}_{R}$ a class of right $R$-modules, $\mathcal{D}_{S}$ a class of right $S$-modules with $\left(\mathcal{D}_{S}\right)_{R} \subseteq \mathcal{C}_{R}$ and $\varphi: M_{S} \rightarrow N_{S}$ a right $S$-homomorphism. We show that:

(a) If $\left(\operatorname{Hom}_{R}\left(S, \mathcal{C}_{R}\right)\right)_{S} \subseteq \mathcal{D}_{S}$ and $N_{S} \in \mathcal{D}_{S}$, then $\varphi_{*}: \operatorname{Hom}_{R}(S, M)$ $\rightarrow \operatorname{Hom}_{R}(S, N)$ is a (respectively, special) $\mathcal{D}_{S}$-preenvelope if and only if $\varphi: M_{R} \rightarrow N_{R}$ is a (respectively, special) $\mathcal{C}_{R^{-}}$ preenvelope.

(b) If $\left(\mathcal{C}_{R} \otimes_{R} S\right)_{S} \subseteq \mathcal{D}_{S}$ and $M_{S} \in \mathcal{D}_{S}$, then $\varphi \otimes_{R} 1: M \otimes_{R} S \rightarrow$ $N \otimes_{R} S$ is a (respectively, special) $\mathcal{D}_{S}$-precover if and only if $\varphi: M_{R} \rightarrow N_{R}$ is a (respectively, special) $\mathcal{C}_{R}$-precover.

2. How do the functors $\operatorname{Hom}_{R}(S,-)$ and $\operatorname{Hom}_{R}(-, S)$ behave? We start with the following result.

Theorem 2.1. Let $R \rightarrow S$ be a ring homomorphism, $\mathcal{C}_{R}$ a class of right $R$-modules, $\mathcal{D}_{S}$ a class of right $S$-modules with $\left(\mathcal{D}_{S}\right)_{R} \subseteq \mathcal{C}_{R}$ and 
$\left(\operatorname{Hom}_{R}\left(S, \mathcal{C}_{R}\right)\right)_{S} \subseteq \mathcal{D}_{S}, \varphi: M_{R} \rightarrow N_{R}$ a right $R$-homomorphism with $M_{R} \in \mathcal{C}_{R}$. Consider the following conditions:

(i) $\varphi: M_{R} \rightarrow N_{R}$ is a $\mathcal{C}_{R}$-precover.

(ii) $\varphi_{*}: \operatorname{Hom}_{R}(S, M) \rightarrow \operatorname{Hom}_{R}(S, N)$ is a $\mathcal{D}_{S}$-precover.

(iii) $\varphi_{*}: \operatorname{Hom}_{R}(S, M) \rightarrow \operatorname{Hom}_{R}(S, N)$ is a $\left(\operatorname{Hom}_{R}\left(S, \mathcal{C}_{R}\right)\right)_{S}$-precover.

(iv) $\varphi \varepsilon_{M}: \operatorname{Hom}_{R}(S, M) \rightarrow N_{R}$ is a $\left(\operatorname{Hom}_{R}\left(S, \mathcal{C}_{R}\right)\right)_{R}$-precover.

Then (i) $\Rightarrow$ (ii) $\Rightarrow$ (iii) $\Leftrightarrow$ (iv).

Proof.

(i) $\Rightarrow$ (ii). For any $A_{S} \in \mathcal{D}_{S}$, we have $A_{R} \in \mathcal{C}_{R}$. So we get the exact sequence

$$
\operatorname{Hom}_{R}\left(A \otimes_{S} S, M\right) \longrightarrow \operatorname{Hom}_{R}\left(A \otimes_{S} S, N\right) \longrightarrow 0
$$

Consider the following commutative diagram:

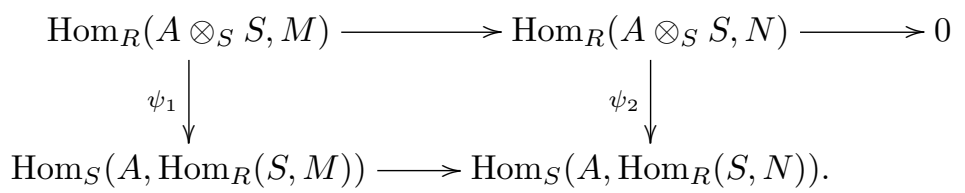

Since $\psi_{1}$ and $\psi_{2}$ are standard isomorphisms, we have the exact sequence

$$
\operatorname{Hom}_{S}\left(A, \operatorname{Hom}_{R}(S, M)\right) \longrightarrow \operatorname{Hom}_{S}\left(A, \operatorname{Hom}_{R}(S, N)\right) \longrightarrow 0
$$

Thus, $\varphi_{*}: \operatorname{Hom}_{R}(S, M) \rightarrow \operatorname{Hom}_{R}(S, N)$ is a $\mathcal{D}_{S \text {-precover since }}$ $\operatorname{Hom}_{R}(S, M) \in \mathcal{D}_{S}$.

(ii) $\Rightarrow$ (iii) is obvious since $\left(\operatorname{Hom}_{R}\left(S, \mathcal{C}_{R}\right)\right)_{S} \subseteq \mathcal{D}_{S}$.

(iii) $\Rightarrow$ (iv). Let $G_{R} \in \mathcal{C}_{R}$ and $\zeta: \operatorname{Hom}_{R}(S, G) \rightarrow N_{R}$ be any $R$-homomorphism. Since $\left(\operatorname{Hom}_{R}(S, G)\right)_{R} \in \mathcal{C}_{R}$, there exists $\sigma: \operatorname{Hom}_{R}\left(S, \operatorname{Hom}_{R}(S, G)\right) \rightarrow \operatorname{Hom}_{R}(S, M)$ such that the following 
diagram is commutative:

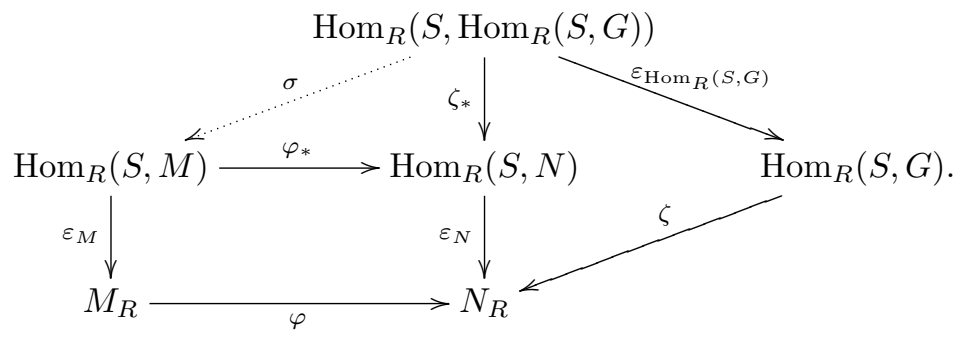

So we have

$$
\begin{aligned}
\left(\varphi \varepsilon_{M}\right)\left(\sigma \eta_{\operatorname{Hom}_{R}(S, G)}\right) & =\varepsilon_{N}\left(\varphi_{*} \sigma\right) \eta_{\operatorname{Hom}_{R}(S, G)}=\varepsilon_{N} \zeta_{*} \eta_{\operatorname{Hom}_{R}(S, G)} \\
& =\zeta \varepsilon_{\operatorname{Hom}_{R}(S, G)} \eta_{\operatorname{Hom}_{R}(S, G)}=\zeta .
\end{aligned}
$$

Thus, $\varphi \varepsilon_{M}: \operatorname{Hom}_{R}(S, M) \rightarrow N_{R}$ is a $\left(\operatorname{Hom}_{R}\left(S, \mathcal{C}_{R}\right)\right)_{R}$-precover.

(iv) $\Rightarrow$ (iii). For any $F_{R} \in \mathcal{C}_{R}$, by (iv), we get the exact sequence

$\operatorname{Hom}_{R}\left(\operatorname{Hom}_{R}(S, F), \operatorname{Hom}_{R}(S, M)\right) \longrightarrow \operatorname{Hom}_{R}\left(\operatorname{Hom}_{R}(S, F), N\right) \longrightarrow 0$.

Observe the following commutative diagram:

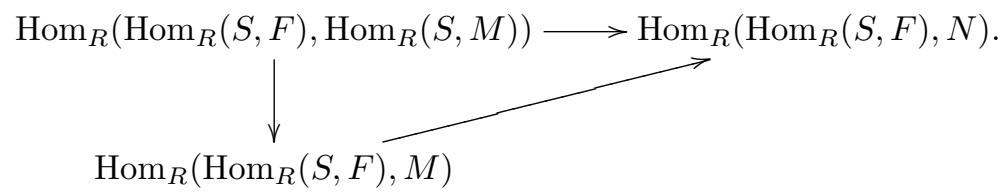

Then we obtain the exact sequence

$$
\operatorname{Hom}_{R}\left(\operatorname{Hom}_{R}(S, F), M\right) \longrightarrow \operatorname{Hom}_{R}\left(\operatorname{Hom}_{R}(S, F), N\right) \longrightarrow 0 .
$$

Consider the following commutative diagram:

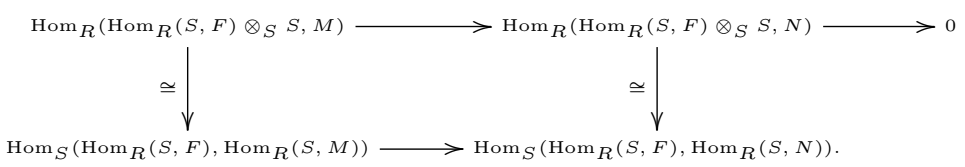

Then we get the exact sequence

$\operatorname{Hom}_{S}\left(\operatorname{Hom}_{R}(S, F), \operatorname{Hom}_{R}(S, M)\right) \rightarrow \operatorname{Hom}_{S}\left(\operatorname{Hom}_{R}(S, F), \operatorname{Hom}_{R}(S, N)\right) \rightarrow 0$.

So $\operatorname{Hom}_{R}(S, M) \rightarrow \operatorname{Hom}_{R}(S, N)$ is a $\left(\operatorname{Hom}_{R}\left(S, \mathcal{C}_{R}\right)\right)_{S}$-precover. 
Corollary 2.2. Let $R \rightarrow S$ be a ring homomorphism, $\mathcal{C}_{R}$ a class of right $R$-modules, $\mathcal{D}_{S}$ a class of right $S$-modules with $\left(\mathcal{D}_{S}\right)_{R} \subseteq \mathcal{C}_{R}$ and $\left(\operatorname{Hom}_{R}\left(S, \mathcal{C}_{R}\right)\right)_{S} \subseteq \mathcal{D}_{S}, \varphi: M_{R} \rightarrow N_{R}$ a right $R$-homomorphism with $M_{R} \in \mathcal{C}_{R}$ and $\varepsilon_{M}$ an isomorphism. Consider the following conditions:

(i) $\varphi: M_{R} \rightarrow N_{R}$ is a $\mathcal{C}_{R}$-cover.

(ii) $\varphi_{*}: \operatorname{Hom}_{R}(S, M) \rightarrow \operatorname{Hom}_{R}(S, N)$ is a $\mathcal{D}_{S}$-cover.

(iii) $\varphi_{*}: \operatorname{Hom}_{R}(S, M) \rightarrow \operatorname{Hom}_{R}(S, N)$ is a $\left(\operatorname{Hom}_{R}\left(S, \mathcal{C}_{R}\right)\right)_{S}$-cover.

(iv) $\varphi \varepsilon_{M}: \operatorname{Hom}_{R}(S, M) \rightarrow N_{R}$ is a $\left(\operatorname{Hom}_{R}\left(S, \mathcal{C}_{R}\right)\right)_{R}$-cover.

Then (i) $\Rightarrow$ (ii) $\Rightarrow$ (iii) $\Leftrightarrow$ (iv).

Proof.

(i) $\Rightarrow$ (ii). If the $S$-homomorphism $\chi: \operatorname{Hom}_{R}(S, M) \rightarrow \operatorname{Hom}_{R}(S, M)$ satisfies $\varphi_{*} \chi=\varphi_{*}$, then $\varphi\left(\varepsilon_{M} \chi \varepsilon_{M}^{-1}\right)=\varepsilon_{N} \varphi_{*} \chi \varepsilon_{M}^{-1}=\varepsilon_{N} \varphi_{*} \varepsilon_{M}^{-1}=$ $\varphi \varepsilon_{M} \varepsilon_{M}^{-1}=\varphi$. Thus, $\varepsilon_{M} \chi \varepsilon_{M}^{-1}$ is an isomorphism, and so is $\chi$. Therefore,

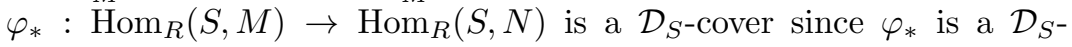
precover by Theorem 2.1 .

(ii) $\Rightarrow$ (iii) is evident.

(iii) $\Rightarrow$ (iv). If the $R$-homomorphism $\omega: \operatorname{Hom}_{R}(S, M) \rightarrow \operatorname{Hom}_{R}(S, M)$ satisfies $\varphi \varepsilon_{M} \omega=\varphi$, then $\varphi_{*}\left(\varepsilon_{M}\right)_{*} \omega_{*}=\varphi_{*}$. Thus, $\left(\varepsilon_{M}\right)_{*} \omega_{*}$ is an isomorphism by (iii). So $\omega=\varepsilon_{M} \omega_{*} \varepsilon_{M}^{-1}$ is an isomorphism. We see that $\varphi \varepsilon_{M}: M_{R} \rightarrow N_{R}$ is a $\left(\operatorname{Hom}_{R}\left(S, \mathcal{C}_{R}\right)\right)_{R}$-cover since $\varphi \varepsilon_{M}$ is a $\left(\operatorname{Hom}_{R}\left(S, \mathcal{C}_{R}\right)\right)_{R}$-precover by Theorem 2.1 .

(iv) $\Rightarrow$ (iii). Let the $S$-homomorphism $\xi: \operatorname{Hom}_{R}(S, M) \rightarrow$ $\operatorname{Hom}_{R}(S, M)$ satisfy $\varphi_{*} \xi=\varphi_{*}$. Then $\varphi \varepsilon_{M} \xi=\varepsilon_{N} \varphi_{*} \xi=\varepsilon_{N} \varphi_{*}=\varphi \varepsilon_{M}$. Thus, $\xi$ is an isomorphism, and so $\varphi_{*}: \operatorname{Hom}_{R}(S, M) \rightarrow \operatorname{Hom}_{R}(S, N)$ is a $\left(\operatorname{Hom}_{R}\left(S, \mathcal{C}_{R}\right)\right)_{S}$-cover by Theorem 2.1 .

Theorem 2.3. Let $R \rightarrow S$ be a ring homomorphism, $\mathcal{C}_{R}$ a class of right $R$-modules, $\mathcal{D}_{S}$ a class of right $S$-modules with $\left(\mathcal{D}_{S}\right)_{R} \subseteq \mathcal{C}_{R}$ and $\left(\operatorname{Hom}_{R}\left(S, \mathcal{C}_{R}\right)\right)_{S} \subseteq \mathcal{D}_{S}, \varphi: M_{S} \rightarrow N_{S}$ a right $S$-homomorphism with $N_{S} \in \mathcal{D}_{S}$. Consider the following conditions:

(i) $\varphi_{*}: \operatorname{Hom}_{R}(S, M) \rightarrow \operatorname{Hom}_{R}(S, N)$ is a $\mathcal{D}_{S}$-preenvelope.

(ii) $\varphi_{*} \eta_{M}: M_{S} \rightarrow \operatorname{Hom}_{R}(S, N)$ is a $\left(\operatorname{Hom}_{R}\left(S, \mathcal{C}_{R}\right)\right)_{S}$-preenvelope.

(iii) $\varphi: M_{R} \rightarrow N_{R}$ is a $\mathcal{C}_{R}$-preenvelope.

Then (i) $\Rightarrow$ (ii) $\Leftrightarrow$ (iii). 
Proof.

(i) $\Rightarrow$ (ii). For any $H_{R} \in \mathcal{C}_{R}, \operatorname{Hom}_{R}\left(S, \operatorname{Hom}_{R}(S, H)\right) \in \mathcal{D}_{S}$ by hypothesis. So, for any $S$-homomorphism $\theta: M_{S} \rightarrow \operatorname{Hom}_{R}(S, H)$, there is an $S$-homomorphism $\xi: \operatorname{Hom}_{R}(S, N) \rightarrow \operatorname{Hom}_{R}\left(S, \operatorname{Hom}_{R}(S, H)\right)$ such that the following diagram commutes.

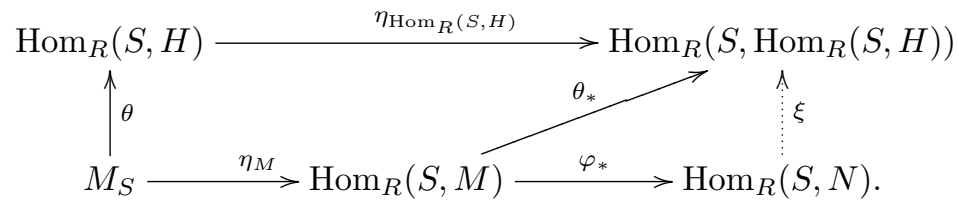

Hence, we obtain $\left(\varepsilon_{H}\right)_{*} \xi\left(\varphi_{*} \eta_{M}\right)=\left(\varepsilon_{H}\right)_{*} \theta_{*} \eta_{M}=\left(\varepsilon_{H}\right)_{*} \eta_{\mathrm{Hom}_{R}(S, H)} \theta=$ $\theta$. Thus, $\varphi_{*} \eta_{M}: M_{S} \rightarrow \operatorname{Hom}_{R}(S, N)$ is a $\left(\operatorname{Hom}_{R}\left(S, \mathcal{C}_{R}\right)\right)_{S}$-preenvelope.

(ii) $\Rightarrow$ (iii). For any $G_{R} \in \mathcal{C}_{R}$ and any $R$-homomorphism $f: M_{R} \rightarrow$ $G_{R}$, by (ii), there exists an $S$-homomorphism $\gamma: \operatorname{Hom}_{R}(S, N) \rightarrow$ $\operatorname{Hom}_{R}(S, G)$ such that the following diagram commutes.

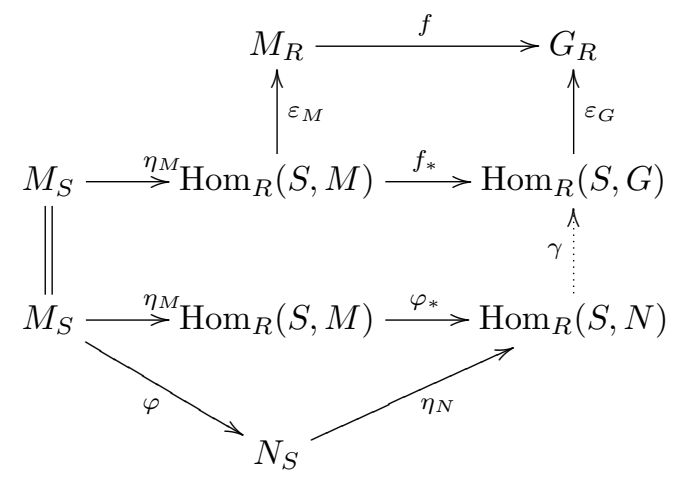

So we have

$$
\left(\varepsilon_{G} \gamma \eta_{N}\right) \varphi=\varepsilon_{G} \gamma \varphi_{*} \eta_{M}=\varepsilon_{G} f_{*} \eta_{M}=f \varepsilon_{M} \eta_{M}=f
$$

Thus, $\varphi: M_{R} \rightarrow N_{R}$ is a $\mathcal{C}_{R}$-preenvelope.

(iii) $\Rightarrow$ (ii). For any $H_{R} \in \mathcal{C}_{R}$ and any $S$-homomorphism $\theta: M_{S} \rightarrow$ $\operatorname{Hom}_{R}(S, H)$, by (3), there exists an $R$-homomorphism $\psi: N_{R} \rightarrow$ $\operatorname{Hom}_{R}(S, H)$ such that $\psi \varphi=\theta$. 
Consider the following commutative diagram:

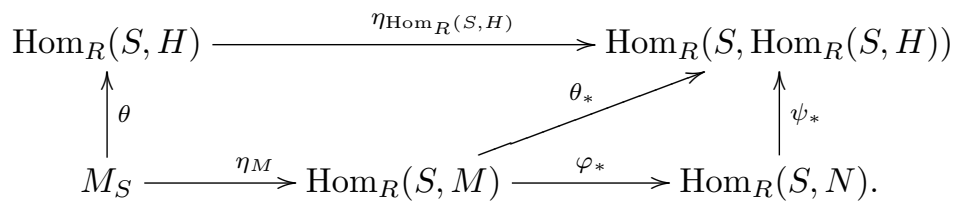

It follows that $\left(\left(\varepsilon_{H}\right)_{*} \psi_{*}\right)\left(\varphi_{*} \eta_{M}\right)=\left(\varepsilon_{H}\right)_{*} \theta_{*} \eta_{M}=\left(\varepsilon_{H}\right)_{*} \eta_{\mathrm{Hom}_{R}(S, H)} \theta=$ $\theta$. Therefore, $\varphi_{*} \eta_{M}: M_{S} \rightarrow \operatorname{Hom}_{R}(S, N)$ is a $\left(\operatorname{Hom}_{R}\left(S, \mathcal{C}_{R}\right)\right)_{S^{-}}$ preenvelope.

Corollary 2.4. Let $R \rightarrow S$ be a ring homomorphism, $\mathcal{C}_{R}$ a class of right $R$-modules, $\mathcal{D}_{S}$ a class of right $S$-modules with $\left(\mathcal{D}_{S}\right)_{R} \subseteq \mathcal{C}_{R}$ and $\left(\operatorname{Hom}_{R}\left(S, \mathcal{C}_{R}\right)\right)_{S} \subseteq \mathcal{D}_{S}, \varphi: M_{S} \rightarrow N_{S}$ a right $S$-homomorphism with $N_{S} \in \mathcal{D}_{S}$ and $\varepsilon_{N}$ an isomorphism. Consider the following conditions:

(i) $\varphi_{*}: \operatorname{Hom}_{R}(S, M) \rightarrow \operatorname{Hom}_{R}(S, N)$ is a $\mathcal{D}_{S}$-envelope.

(ii) $\varphi_{*} \eta_{M}: M_{S} \rightarrow \operatorname{Hom}_{R}(S, N)$ is a $\left(\operatorname{Hom}_{R}\left(S, \mathcal{C}_{R}\right)\right)_{S}$-envelope.

(iii) $\varphi: M_{R} \rightarrow N_{R}$ is a $\mathcal{C}_{R}$-envelope.

Then (i) $\Rightarrow$ (ii) $\Leftrightarrow$ (iii).

Proof. Since $\varepsilon_{N}$ is an $R$-isomorphism and $\varepsilon_{N} \eta_{N}=1$, we have $\eta_{N}$ is an $S$-isomorphism, and so $\varepsilon_{N}=\eta_{N}^{-1}$ is also an $S$-isomorphism. We claim that any $R$-homomorphism from $N$ to $N$ is also an $S$ homomorphism. In fact, let $\alpha: N \rightarrow N$ be any $R$-homomorphism and $\alpha_{*}: \operatorname{Hom}_{R}(S, N) \rightarrow \operatorname{Hom}_{R}(S, N)$ the induced $S$-homomorphism. Then $\alpha=\varepsilon_{N} \alpha_{*} \varepsilon_{N}^{-1}$ is an $S$-homomorphism. Thus, the result can be easily deduced from Theorem 2.3.

Corollary 2.5. Let $R \rightarrow S$ be a ring homomorphism with ${ }_{R} S$ flat.

(i) [5, page 125, Exercise 5.4.4]. If $\varphi: M_{R} \rightarrow N_{R}$ is an injective precover of $R$-modules, then $\varphi_{*}: \operatorname{Hom}_{R}(S, M) \rightarrow \operatorname{Hom}_{R}(S, N)$ is an injective precover of $S$-modules.

(ii) If $\varphi: M_{S} \rightarrow N_{S}$ a right $S$-homomorphism such that $N_{S}$ is injective and $\varphi_{*}: \operatorname{Hom}_{R}(S, M) \rightarrow \operatorname{Hom}_{R}(S, N)$ is an injective preenvelope of $S$-modules, then $\varphi: M_{R} \rightarrow N_{R}$ is an injective preenvelope of $R$-modules. 
Proof. Since any injective right $S$-module is an injective right $R$ module by [7, Corollary 3.6A] and $\operatorname{Hom}_{R}(S, E)$ is an injective right $S$-module for any injective right $R$-module $E$ by [7, Corollary 3.6B], the result holds by Theorems 2.1 and 2.3.

Recall that a right $R$-module $M$ is cotorsion [5, Definition 5.3.22] if $\operatorname{Ext}_{R}^{1}(F, M)=0$ for any flat right $R$-module $F$. It is known that every right $R$-module over any ring $R$ has a cotorsion envelope by [5, Theorem 7.4.4] and [13, Theorem 3.4.6].

Lemma 2.6. Let $R \rightarrow S$ be a ring homomorphism.

(i) If ${ }_{R} S$ is flat, then any cotorsion right $S$-module is a cotorsion right $R$-module.

(ii) If $S_{R}$ is flat, then $\operatorname{Hom}_{R}(S, A)$ is a cotorsion right $S$-module for any cotorsion right $R$-module $A_{R}$.

Proof.

(i) Let $N_{S}$ be a cotorsion right $S$-module and $A_{R}$ a flat right $R$ module. Then $A \otimes_{R} S$ is a flat right $S$-module. From [10, Theorem 11.65], we have $\operatorname{Ext}_{R}^{1}(A, N) \cong \operatorname{Ext}_{S}^{1}\left(A \otimes_{R} S, N\right)=0$. So $N_{R}$ is a cotorsion right $R$-module.

(ii) If $B_{S}$ is a flat right $S$-module, then $B_{R}$ is a flat right $R$-module. There is an exact sequence $0 \rightarrow K_{S} \rightarrow P_{S} \rightarrow B_{S} \rightarrow 0$ of right $S$-modules with $P_{S}$ projective. Consider the following commutative diagram with exact rows:

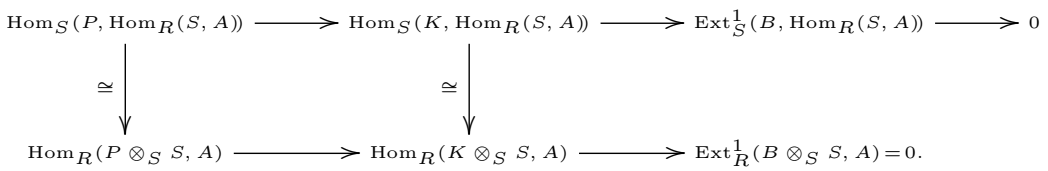

So $\operatorname{Ext}_{S}^{1}\left(B, \operatorname{Hom}_{R}(S, A)\right)=0$. Hence, $\operatorname{Hom}_{R}(S, A)$ is a cotorsion right $S$-module.

Proposition 2.7. Let $R \rightarrow S$ be a ring homomorphism with ${ }_{R} S$ and $S_{R}$ flat. 
(i) If $\varphi: M_{R} \rightarrow N_{R}$ is a cotorsion precover of $R$-modules, then $\varphi_{*}: \operatorname{Hom}_{R}(S, M) \rightarrow \operatorname{Hom}_{R}(S, N)$ is a cotorsion precover of $S$ modules.

(ii) If $\varphi: M_{S} \rightarrow N_{S}$ is a right $S$-homomorphism such that $N_{S}$ is cotorsion and $\varphi_{*}: \operatorname{Hom}_{R}(S, M) \rightarrow \operatorname{Hom}_{R}(S, N)$ is a cotorsion preenvelope of $S$-modules, then $\varphi: M_{R} \rightarrow N_{R}$ is a cotorsion preenvelope of $R$-modules.

Proof. It follows from Theorems 2.1, 2.3 and Lemma 2.6.

We next turn to the special precovers and preenvelopes under the change of rings.

Let $\mathcal{C}$ be a class of right $R$-modules. According to [5, Definition 7.1.6] or [6, Definition 2.1.12], a $\mathcal{C}$-precover $f: M \rightarrow N$ is called special if $f$ is an epimorphism and $\operatorname{ker}(f) \in \mathcal{C}^{\perp}=\left\{X: \operatorname{Ext}_{R}^{1}(C, X)=\right.$ 0 for all $C \in \mathcal{C}\}$. Dually, a $\mathcal{C}$-preenvelope $g: M \rightarrow N$ is called special if $g$ is a monomorphism and $\operatorname{coker}(g) \in{ }^{\perp} \mathcal{C}=\left\{X: \operatorname{Ext}_{R}^{1}(X, C)=\right.$ 0 for all $C \in \mathcal{C}\}$.

Proposition 2.8. Let $R \rightarrow S$ be a ring homomorphism with $S_{R}$ projective, $\mathcal{C}_{R}$ a class of right $R$-modules, $\mathcal{D}_{S}$ a class of right $S$-modules with $\left(\mathcal{D}_{S}\right)_{R} \subseteq \mathcal{C}_{R}$ and $\left(\operatorname{Hom}_{R}\left(S, \mathcal{C}_{R}\right)\right)_{S} \subseteq \mathcal{D}_{S}, \varphi: M_{S} \rightarrow N_{S}$ a right S-homomorphism.

(i) If $\varphi: M_{R} \rightarrow N_{R}$ is a special $\mathcal{C}_{R}$-precover, then $\varphi_{*}: \operatorname{Hom}_{R}(S, M) \rightarrow$ $\operatorname{Hom}_{R}(S, N)$ is a special $\mathcal{D}_{S}$-precover.

(ii) If $\varphi: M_{S} \rightarrow N_{S}$ is a special $\mathcal{D}_{S}$-preenvelope, then $\varphi: M_{R} \rightarrow N_{R}$ is a special $\mathcal{C}_{R}$-preenvelope.

Proof.

(i) By hypothesis, $\varphi$ is epic. Thus, there is an exact sequence $0 \rightarrow K_{S} \rightarrow M_{S} \rightarrow N_{S} \rightarrow 0$ of right $S$-modules, which induces the exact sequence $0 \rightarrow K_{R} \rightarrow M_{R} \rightarrow N_{R} \rightarrow 0$ of right $R$-modules with $M_{R} \in \mathcal{C}_{R}$ and $K_{R} \in \mathcal{C}_{R}^{\perp}$.

Since $S_{R}$ is projective, we get the exact sequence

$$
0 \longrightarrow \operatorname{Hom}_{R}(S, K) \longrightarrow \operatorname{Hom}_{R}(S, M) \longrightarrow \operatorname{Hom}_{R}(S, N) \longrightarrow 0
$$

of right $S$-modules with $\operatorname{Hom}_{R}(S, M) \in \mathcal{D}_{S}$. 
For any $B_{S} \in \mathcal{D}_{S}$, we have $B_{R} \in \mathcal{C}_{R}$. Thus, $\operatorname{Ext}_{S}^{1}\left(B, \operatorname{Hom}_{R}(S, K)\right) \cong$ $\operatorname{Ext}_{R}^{1}(B, K)=0$ by [10, Theorem 11.66]. Hence, $\operatorname{Hom}_{R}(S, K) \in \mathcal{D} \frac{\perp}{S}$, and so $\varphi_{*}: \operatorname{Hom}_{R}(S, M) \rightarrow \operatorname{Hom}_{R}(S, N)$ is a special $\mathcal{D}_{S}$-precover.

(ii) There is an exact sequence $0 \rightarrow M_{S} \rightarrow N_{S} \rightarrow L_{S} \rightarrow 0$ of right $S$-modules with $N_{S} \in \mathcal{D}_{S}$ and $L_{S} \in{ }^{\perp} \mathcal{D}_{S}$. So we get the exact sequence $0 \rightarrow M_{R} \rightarrow N_{R} \rightarrow L_{R} \rightarrow 0$ of right $R$-modules with $N_{R} \in \mathcal{C}_{R}$.

For any $A_{R} \in \mathcal{C}_{R}, \operatorname{Hom}_{R}\left(S, A_{R}\right) \in \mathcal{D}_{S}$. Utilizing [10, Theorem 11.66], we get $\operatorname{Ext}_{R}^{1}(L, A) \cong \operatorname{Ext}_{S}^{1}\left(L, \operatorname{Hom}_{R}(S, A)\right)=0$. Thus, $L_{R} \in$ ${ }^{\perp} \mathcal{C}_{R}$, and so $\varphi: M_{R} \rightarrow N_{R}$ is a special $\mathcal{C}_{R}$-preenvelope.

At the end of this section, we point out that the functor $\operatorname{Hom}_{R}(-, S)$ converts a (pre)envelope into a (pre)cover under some conditions. The proofs of the next results are essentially dual to those of Theorem 2.1 and Corollary 2.2, and so we leave the proofs to the reader.

Theorem 2.9. Let $R \rightarrow S$ be a ring homomorphism, $\mathcal{C}_{R}$ a class of right $R$-modules, ${ }_{S} \mathcal{D}$ a class of left $S$-modules with $\left(\mathcal{C}_{R}\right)^{*} \subseteq{ }_{S} \mathcal{D}$ and $\left({ }_{S} \mathcal{D}\right)^{*} \subseteq \mathcal{C}_{R}, \varphi: M_{R} \rightarrow N_{R}$ a right $R$-homomorphism with $N_{R} \in \mathcal{C}_{R}$. Consider the following conditions:

(i) $\varphi: M_{R} \rightarrow N_{R}$ is a $\mathcal{C}_{R}$-preenvelope.

(ii) $\varphi^{*}: N^{*} \rightarrow M^{*}$ is a ${ }_{S} \mathcal{D}$-precover.

(iii) $\varphi^{*}: N^{*} \rightarrow M^{*}$ is a $\left(\mathcal{C}_{R}\right)^{*}$-precover.

(iv) $\delta_{N} \varphi: M_{R} \rightarrow N^{* *}$ is a $\left(\mathcal{C}_{R}\right)^{* *}$-preenvelope.

Then (i) $\Rightarrow$ (ii) $\Rightarrow$ (iii) $\Leftrightarrow$ (iv).

Corollary 2.10. Let $R \rightarrow S$ be a ring homomorphism, $\mathcal{C}_{R}$ a class of right $R$-modules, ${ }_{S} \mathcal{D}$ a class of left $S$-modules with $\left(\mathcal{C}_{R}\right)^{*} \subseteq{ }_{S} \mathcal{D}$ and $\left({ }_{S} \mathcal{D}\right)^{*} \subseteq \mathcal{C}_{R}, \varphi: M_{R} \rightarrow N_{R}$ a right $R$-homomorphism with $N_{R}$ $S$-reflexive. Consider the following conditions:

(i) $\varphi: M_{R} \rightarrow N_{R}$ is a $\mathcal{C}_{R}$-envelope.

(ii) $\varphi^{*}: N^{*} \rightarrow M^{*}$ is a ${ }_{S} \mathcal{D}$-cover.

(iii) $\varphi^{*}: N^{*} \rightarrow M^{*}$ is a $\left(\mathcal{C}_{R}\right)^{*}$-cover.

(iv) $\delta_{N} \varphi: M_{R} \rightarrow N^{* *}$ is a $\left(\mathcal{C}_{R}\right)^{* *}$-envelope.

Then (i) $\Rightarrow$ (ii) $\Rightarrow$ (iii) $\Leftrightarrow$ (iv). 
3. How does the functor $-\otimes_{R} S$ behave? By proofs analogous to the proofs of Theorem 2.1 and Corollary 2.2, one can prove that the functor $-\otimes_{R} S$ preserves (pre)envelopes under some conditions as follows.

Theorem 3.1. Let $\varphi: R \rightarrow S$ be a ring homomorphism, $\mathcal{C}_{R}$ a class of right $R$-modules, $\mathcal{D}_{S}$ a class of right $S$-modules with $\left(\mathcal{D}_{S}\right)_{R} \subseteq \mathcal{C}_{R}$ and $\left(\mathcal{C}_{R} \otimes_{R} S\right)_{S} \subseteq \mathcal{D}_{S}, \varphi: M_{R} \rightarrow N_{R}$ a right $R$-homomorphism with $N_{R} \in \mathcal{C}_{R}$. Consider the following conditions:

(i) $\varphi: M_{R} \rightarrow N_{R}$ is a $\mathcal{C}_{R}$-preenvelope.

(ii) $\varphi \otimes_{R} 1: M \otimes_{R} S \rightarrow N \otimes_{R} S$ is a $\mathcal{D}_{S}$-preenvelope.

(iii) $\varphi \otimes_{R} 1: M \otimes_{R} S \rightarrow N \otimes_{R} S$ is a $\left(\mathcal{C}_{R} \otimes_{R} S\right)_{S}$-preenvelope.

(iv) $\nu_{N} \varphi: M_{R} \rightarrow N \otimes_{R} S$ is a $\left(\mathcal{C}_{R} \otimes_{R} S\right)_{R}$-preenvelope.

Then (i) $\Rightarrow$ (ii) $\Rightarrow$ (iii) $\Leftrightarrow$ (iv).

Corollary 3.2. Let $\varphi: R \rightarrow S$ be a ring homomorphism, $\mathcal{C}_{R}$ a class of right $R$-modules, $\mathcal{D}_{S}$ a class of right $S$-modules with $\left(\mathcal{D}_{S}\right)_{R} \subseteq \mathcal{C}_{R}$ and $\left(\mathcal{C}_{R} \otimes_{R} S\right)_{S} \subseteq \mathcal{D}_{S}, \varphi: M_{R} \rightarrow N_{R}$ a right $R$-homomorphism with $N_{R} \in \mathcal{C}_{R}$ and $\nu_{N}$ an isomorphism. Consider the following conditions:

(i) $\varphi: M_{R} \rightarrow N_{R}$ is a $\mathcal{C}_{R}$-envelope.

(ii) $\varphi \otimes_{R} 1: M \otimes_{R} S \rightarrow N \otimes_{R} S$ is a $\mathcal{D}_{S}$-envelope.

(iii) $\varphi \otimes_{R} 1: M \otimes_{R} S \rightarrow N \otimes_{R} S$ is a $\left(\mathcal{C}_{R} \otimes_{R} S\right)_{S}$-envelope.

(iv) $\varphi: M_{R} \rightarrow N_{R}$ is a $\left(\mathcal{C}_{R} \otimes_{R} S\right)_{R}$-envelope.

Then (i) $\Rightarrow$ (ii) $\Rightarrow$ (iii) $\Leftrightarrow$ (iv).

Let $S$ be a multiplicative subset of a commutative ring $R$. We can form the ring of fractions $S^{-1} R$. There is a canonical ring homomorphism $R \rightarrow S^{-1} R$. For an $R$-module $M$, we also can construct the localization of $M$ with respect to $S$, denoted by $S^{-1} M$, which is an $S^{-1} R$-module, and hence an $R$-module.

Corollary 3.3. Let $S$ be a multiplicative subset of a commutative ring $R$ and $\varphi: M_{R} \rightarrow N_{R}$ an $R$-homomorphism.

(i) If $\varphi: M_{R} \rightarrow N_{R}$ is a flat preenvelope of $R$-modules, then $S^{-1} \varphi: S^{-1} M \rightarrow S^{-1} N$ is a flat preenvelope of $S^{-1} R$-modules. 
(ii) If $S^{-1} \varphi: S^{-1} M \rightarrow S^{-1} N$ is a flat envelope of $R$-modules, then $S^{-1} \varphi: S^{-1} M \rightarrow S^{-1} N$ is a flat envelope of $S^{-1} R$-modules.

Proof.

(i) Note that any flat $S^{-1} R$-module is a flat $R$-module and $A \otimes_{R}$ $S^{-1} R \cong S^{-1} A$ is a flat $S^{-1} R$-module for any flat $R$-module $A$. Then Theorem 3.1 applies.

(ii) Since $\nu_{S^{-1} N}: S^{-1} N \rightarrow S^{-1} N \otimes_{R} S^{-1} R$ is an isomorphism by [10, Lemma 3.75], the conclusion follows from Corollary 3.2.

Recall that a right $R$-module $M$ is $F P$-injective [11] if $\operatorname{Ext}_{R}^{1}(N, M)=$ 0 for all finitely presented right $R$-modules $N$.

Lemma 3.4. Let $R \rightarrow S$ be a ring homomorphism with ${ }_{R} S$ flat. Then any $F P$-injective right $S$-module is an FP-injective right $R$-module.

Proof. Let $N_{S}$ be an $F P$-injective right $S$-module and $A_{R}$ a finitely presented right $R$-module. Then $A \otimes_{R} S$ is a finitely presented right $S$ module. From [10, Theorem 11.65], $\operatorname{Ext}_{R}^{1}(A, N) \cong \operatorname{Ext}_{S}^{1}\left(A \otimes_{R} S, N\right)=$ 0 . So $N_{R}$ is an $F P$-injective right $R$-module.

It is known that every right $R$-module has an $F P$-injective preenvelope over any ring $R$ by [5, Proposition 6.2.4], but not every right $R$-module has an FP-injective envelope by [6, Corollary 6.3.19].

Recall that $R$ is a right coherent ring if every finitely generated right ideal is finitely presented.

Proposition 3.5. Let $S$ be a multiplicative subset of a commutative coherent ring $R$ and $\varphi: M_{R} \rightarrow N_{R}$ an R-homomorphism.

(i) If $\varphi: M_{R} \rightarrow N_{R}$ is an FP-injective preenvelope of $R$-modules, then $S^{-1} \varphi: S^{-1} M \rightarrow S^{-1} N$ is an FP-injective preenvelope of $S^{-1} R$-modules.

(ii) If $S^{-1} \varphi: S^{-1} M \rightarrow S^{-1} N$ is an FP-injective envelope of $R$ modules, then $S^{-1} \varphi: S^{-1} M \rightarrow S^{-1} N$ is an FP-injective envelope of $S^{-1} R$-modules. 
Proof. By Lemma 3.4, any $F P$-injective $S^{-1} R$-module is an $F P$ injective $R$-module. By [9, Theorem 3.21], $B \otimes S^{-1} R \cong S^{-1} B$ is an $F P$-injective $S^{-1} R$-module for any $F P$-injective $R$-module $B$ since $R$ is a coherent ring. So (i) holds by Theorem 3.1 and (ii) follows from Corollary 3.2 .

Theorem 3.6. Let $\varphi: R \rightarrow S$ be a ring homomorphism, $\mathcal{C}_{R}$ a class of right $R$-modules, $\mathcal{D}_{S}$ a class of right $S$-modules with $\left(\mathcal{D}_{S}\right)_{R} \subseteq \mathcal{C}_{R}$ and $\left(\mathcal{C}_{R} \otimes_{R} S\right)_{S} \subseteq \mathcal{D}_{S}, \varphi: M_{S} \rightarrow N_{S}$ a right $S$-homomorphism with $M_{S} \in \mathcal{D}_{S}$. Consider the following conditions:

(i) $\varphi: M_{S} \rightarrow N_{S}$ is a $\mathcal{D}_{S}$-precover.

(ii) $\mu_{N}\left(\varphi \otimes_{R} 1\right): M \otimes_{R} S \rightarrow N_{S}$ is a $\left(\mathcal{C}_{R} \otimes_{R} S\right)_{S}$-precover.

(iii) $\varphi: M_{R} \rightarrow N_{R}$ is a $\mathcal{C}_{R}$-precover.

Then (i) $\Rightarrow$ (ii) $\Leftrightarrow$ (iii).

Proof.

(i) $\Rightarrow$ (ii). For any $Q_{R} \in \mathcal{C}_{R}$ and any $S$-homomorphism $\alpha: Q \otimes_{R} S \rightarrow$ $N_{S}$, by (i), there exists $\beta: Q \otimes_{R} S \rightarrow M_{S}$ such that the following diagram is commutative.

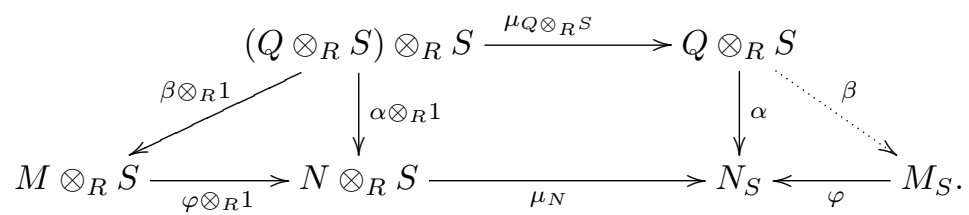

Thus, we obtain $\mu_{N}\left(\varphi \otimes_{R} 1\right)\left(\beta \otimes_{R} 1\right)\left(\nu_{Q} \otimes_{R} 1\right)=\mu_{N}\left(\alpha \otimes_{R} 1\right)\left(\nu_{Q} \otimes_{R} 1\right)=$ $\alpha \mu_{Q \otimes_{R} S}\left(\nu_{Q} \otimes_{R} 1\right)=\alpha$. So $\mu_{N}\left(\varphi \otimes_{R} 1\right): M \otimes_{R} S \rightarrow N_{S}$ is a $\left(\mathcal{C}_{R} \otimes_{R} S\right)_{S^{-}}$ precover.

(ii) $\Rightarrow$ (iii). For any $G_{R} \in \mathcal{C}_{R}$ and any $R$-homomorphism $f: G_{R} \rightarrow$ $N_{R}$, there is an $S$-homomorphism $g: G \otimes_{R} S \rightarrow M \otimes_{R} S$ such that the 
following diagram commutes.

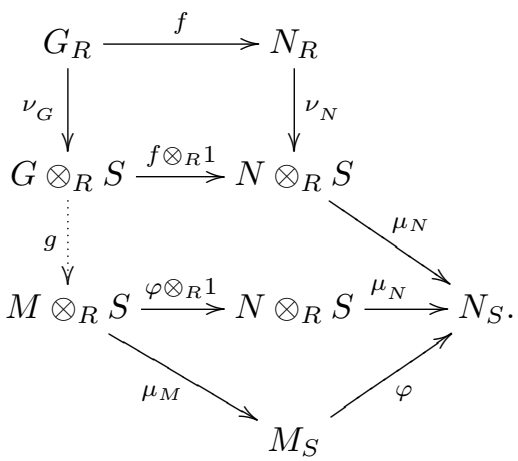

This implies that $\varphi\left(\mu_{M} g \nu_{G}\right)=\mu_{N}\left(\varphi \otimes_{R} 1\right) g \nu_{G}=\mu_{N}\left(f \otimes_{R} 1\right) \nu_{G}=$ $\mu_{N} \nu_{N} f=f$. So $\varphi: M_{R} \rightarrow N_{R}$ is a $\mathcal{C}_{R}$-precover.

The proof of (iii) $\Rightarrow$ (ii) is similar to that of (i) $\Rightarrow$ (ii).

Corollary 3.7. Let $\varphi: R \rightarrow S$ be a ring homomorphism, $\mathcal{C}_{R}$ a class of right $R$-modules, $\mathcal{D}_{S}$ a class of right $S$-modules with $\left(\mathcal{D}_{S}\right)_{R} \subseteq \mathcal{C}_{R}$ and $\left(\mathcal{C}_{R} \otimes_{R} S\right)_{S} \subseteq \mathcal{D}_{S}, \varphi: M_{S} \rightarrow N_{S}$ a right $S$-homomorphism with $M_{S} \in \mathcal{D}_{S}$ and $\nu_{M}$ an isomorphism. Consider the following conditions:

(i) $\varphi: M_{S} \rightarrow N_{S}$ is a $\mathcal{D}_{S}$-cover.

(ii) $\mu_{N}\left(\varphi \otimes_{R} 1\right): M \otimes_{R} S \rightarrow N_{S}$ is a $\left(\mathcal{C}_{R} \otimes_{R} S\right)_{S}$-cover.

(iii) $\varphi: M_{R} \rightarrow N_{R}$ is a $\mathcal{C}_{R}$-cover.

Then (i) $\Rightarrow$ (ii) $\Leftrightarrow$ (iii).

Proof. Since $\nu_{M}$ is an isomorphism and $\mu_{M} \nu_{M}=1$, we have $\nu_{M}$ is an $S$-isomorphism. It is easy to see that any $R$-homomorphism from $M$ to $M$ is also an $S$-homomorphism, and so the result is a consequence of Theorem 3.6.

It is known that every right $R$-module over any ring $R$ has a flat cover by [5, Theorem 7.4.4] and every right $R$-module over a right coherent ring $R$ has an FP-injective cover by [9, Theorem 4.9] and the remark following it. 
Corollary 3.8. Let $S$ be a multiplicative subset of a commutative ring $R$ and $\varphi: M_{R} \rightarrow N_{R}$ an R-homomorphism.

(i) $S^{-1} \varphi: S^{-1} M \rightarrow S^{-1} N$ is a flat precover (respectively, flat cover) of $R$-modules if and only if $S^{-1} \varphi: S^{-1} M \rightarrow S^{-1} N$ is a flat precover (respectively, flat cover) of $S^{-1} R$-modules.

(ii) If $R$ is coherent, then $S^{-1} \varphi: S^{-1} M \rightarrow S^{-1} N$ is an FP-injective precover (respectively, FP-injective cover) of $R$-modules if and only if $S^{-1} \varphi: S^{-1} M \rightarrow S^{-1} N$ is an FP-injective precover (respectively, FP-injective cover) of $S^{-1} R$-modules.

Proof. This is a direct consequence of Theorem 3.6 together with Corollary 3.7 .

Proposition 3.9. Let $R \rightarrow S$ be a ring homomorphism with ${ }_{R} S$ flat, $\mathcal{C}_{R}$ a class of right $R$-modules, $\mathcal{D}_{S}$ a class of right $S$-modules with $\left(\mathcal{D}_{S}\right)_{R} \subseteq \mathcal{C}_{R}$ and $\left(\mathcal{C}_{R} \otimes_{R} S\right)_{S} \subseteq \mathcal{D}_{S}, \varphi: M_{S} \rightarrow N_{S}$ a right $S$ homomorphism.

(i) If $\varphi: M_{S} \rightarrow N_{S}$ is a special $\mathcal{D}_{S}$-precover, then $\varphi: M_{R} \rightarrow N_{R}$ is a special $\mathcal{C}_{R}$-precover.

(ii) If $\varphi: M_{R} \rightarrow N_{R}$ is a special $\mathcal{C}_{R}$-preenvelope, then $\varphi \otimes_{R} 1$ : $M \otimes_{R} S \rightarrow N \otimes_{R} S$ is a special $\mathcal{D}_{S}$-preenvelope.

Proof.

(i) There is an exact sequence $0 \rightarrow K_{S} \rightarrow M_{S} \rightarrow N_{S} \rightarrow 0$ of right $S$-modules with $M_{S} \in \mathcal{D}_{S}$ and $K_{S} \in \mathcal{D}_{S}^{\perp}$. Hence, we get the exact sequence $0 \rightarrow K_{R} \rightarrow M_{R} \rightarrow N_{R} \rightarrow 0$ of right $R$-modules with $M_{R} \in \mathcal{C}_{R}$.

For any $A_{R} \in \mathcal{C}_{R}, A \otimes_{R} S \in \mathcal{D}_{S}$. By [10, Theorem 11.65], we have $\operatorname{Ext}_{R}^{1}(A, K) \cong \operatorname{Ext}_{S}^{1}\left(A \otimes_{R} S, K\right)=0$. So $K_{R} \in \mathcal{C}_{R}^{\perp}$. Thus $\varphi: M_{R} \rightarrow N_{R}$ is a special $\mathcal{C}_{R}$-precover.

(ii) By hypothesis, $\varphi$ is monic. Thus, there is an exact sequence $0 \rightarrow M_{S} \rightarrow N_{S} \rightarrow L_{S} \rightarrow 0$ of right $S$-modules, which gives rise to the exactness of the sequence $0 \rightarrow M_{R} \rightarrow N_{R} \rightarrow L_{R} \rightarrow 0$ of right $R$-modules with $N_{R} \in \mathcal{C}_{R}$ and $L_{R} \in{ }^{\perp} \mathcal{C}_{R}$.

Since ${ }_{R} S$ is flat, we obtain the exact sequence $0 \rightarrow M \otimes_{R} S \rightarrow$ $N \otimes_{R} S \rightarrow L \otimes_{R} S \rightarrow 0$ of right $S$-modules with $N \otimes_{R} S \in \mathcal{D}_{S}$. 
For any $B_{S} \in \mathcal{D}_{S}, B_{R} \in \mathcal{C}_{R}$. By [10, Theorem 11.65], we have $\operatorname{Ext}_{S}^{1}\left(L \otimes_{R} S, B\right) \cong \operatorname{Ext}_{R}^{1}(L, B)=0$. Hence, $L \otimes_{R} S \in{ }^{\perp} \mathcal{D}_{S}$, and so $\varphi \otimes_{R} 1: M \otimes_{R} S \rightarrow N \otimes_{R} S$ is a special $\mathcal{D}_{S}$-preenvelope.

4. Special ring homomorphisms. In this section, we apply the results of the previous sections to study the properties of (pre)covers and (pre)envelopes under some special ring homomorphisms.

We first discuss the (pre)covers and (pre)envelopes under surjective ring homomorphisms. The following lemma is needed.

Lemma 4.1. Let $R \rightarrow S$ be a surjective ring homomorphism and $M_{S}$ a right $S$-module. Then $\operatorname{Hom}_{R}(S, M) \cong M_{S} \cong M \otimes_{R} S$.

Proof. It is routine.

Theorem 4.2. Let $R \rightarrow S$ be a surjective ring homomorphism, $\mathcal{C}_{R}$ a class of right $R$-modules, $\mathcal{D}_{S}$ a class of right $S$-modules with $\left(\mathcal{D}_{S}\right)_{R} \subseteq \mathcal{C}_{R}$ and $\left(\operatorname{Hom}_{R}\left(S, \mathcal{C}_{R}\right)\right)_{S} \subseteq \mathcal{D}_{S}, \varphi: M_{S} \rightarrow N_{S}$ a right $S$ homomorphism.

(i) $\varphi: M_{S} \rightarrow N_{S}$ is a $\mathcal{D}_{S}$-preenvelope (respectively, $\mathcal{D}_{S}$-envelope) if and only if $\varphi: M_{R} \rightarrow N_{R}$ is a $\mathcal{C}_{R}$-preenvelope (respectively, $\mathcal{C}_{R^{-} \text {envelope) }}$

(ii) If $\varphi: M_{R} \rightarrow N_{R}$ is a $\mathcal{C}_{R}$-precover (respectively, $\mathcal{C}_{R}$-cover), then $\varphi: M_{S} \rightarrow N_{S}$ is a $\mathcal{D}_{S}$-precover (respectively, $\mathcal{D}_{S}$-cover).

Proof.

(i) follows from Theorem 2.3, Corollary 2.4 and Lemma 4.1.

(ii) holds by Theorem 2.1, Corollary 2.2 and Lemma 4.1.

Immediately, we have

Corollary 4.3. Let $R \rightarrow S$ be a surjective ring homomorphism with ${ }_{R} S$ flat, $\varphi: M_{S} \rightarrow N_{S}$ a right $S$-homomorphism.

(i) $\varphi: M_{S} \rightarrow N_{S}$ is an injective preenvelope (respectively, injective envelope) of $S$-modules if and only if $\varphi: M_{R} \rightarrow N_{R}$ is an injective preenvelope (respectively, injective envelope) of $R$-modules. 
(ii) If $\varphi: M_{R} \rightarrow N_{R}$ is an injective precover (respectively, injective cover) of $R$-modules, then $\varphi: M_{S} \rightarrow N_{S}$ is an injective precover (respectively, injective cover) of $S$-modules.

Corollary 4.4. Let $R \rightarrow S$ be a surjective ring homomorphism with ${ }_{R} S$ flat and $S_{R}$ projective, $\varphi: M_{S} \rightarrow N_{S}$ a right $S$-homomorphism.

(i) $\varphi: M_{S} \rightarrow N_{S}$ is an FP-injective preenvelope (respectively, FPinjective envelope) of $S$-modules if and only if $\varphi: M_{R} \rightarrow N_{R}$ is an FP-injective preenvelope (respectively, FP-injective envelope) of $R$-modules.

(ii) If $\varphi: M_{R} \rightarrow N_{R}$ is an FP-injective precover (respectively, FPinjective cover) of $R$-modules, then $\varphi: M_{S} \rightarrow N_{S}$ is an FPinjective precover (respectively, FP-injective cover) of $S$-modules.

Proof. We first claim that $\operatorname{Hom}_{R}(S, A)$ is an $F P$-injective right $S$ module for any $F P$-injective right $R$-module $A_{R}$. Indeed, if $B_{S}$ is a finitely presented right $S$-module, then $B_{R}$ is a finitely presented right $R$-module. Thus, $\operatorname{Ext}_{S}^{1}\left(B, \operatorname{Hom}_{R}(S, A)\right) \cong \operatorname{Ext}_{R}^{1}(B, A)=0$ by $[\mathbf{1 0}$, Theorem 11.66]. So $\operatorname{Hom}_{R}(S, A)$ is an $F P$-injective right $S$-module.

In addition, any $F P$-injective right $S$-module is an $F P$-injective right $R$-module by Lemma 3.4. So the result is a direct consequence of Theorem 4.2.

Corollary 4.5. Let $R \rightarrow S$ be a surjective ring homomorphism with $S_{R}$ flat, $\varphi: M_{S} \rightarrow N_{S}$ a right $S$-homomorphism.

(i) $\varphi: M_{S} \rightarrow N_{S}$ is a cotorsion preenvelope (respectively, cotorsion envelope) of $S$-modules if and only if $\varphi: M_{R} \rightarrow N_{R}$ is a cotorsion preenvelope (respectively, cotorsion envelope) of $R$-modules.

(ii) If $\varphi: M_{R} \rightarrow N_{R}$ is a cotorsion precover (respectively, cotorsion cover) of $R$-modules, then $\varphi: M_{S} \rightarrow N_{S}$ is a cotorsion precover (respectively, cotorsion cover) of $S$-modules.

Proof. By Lemma 2.6, $\operatorname{Hom}_{R}(S, A)$ is a cotorsion right $S$-module for any cotorsion right $R$-module $A_{R}$. In addition, any cotorsion right $S$-module is a cotorsion right $R$-module by [13, Proposition 3.3.3]. So the result follows from Theorem 4.2. 
Theorem 4.6. Let $R \rightarrow S$ be a surjective ring homomorphism, $\mathcal{C}_{R}$ a class of right $R$-modules, $\mathcal{D}_{S}$ a class of right $S$-modules with $\left(\mathcal{D}_{S}\right)_{R} \subseteq$ $\mathcal{C}_{R}$ and $\left(\mathcal{C}_{R} \otimes_{R} S\right)_{S} \subseteq \mathcal{D}_{S}, \varphi: M_{S} \rightarrow N_{S}$ a right $S$-homomorphism.

(i) $\varphi: M_{S} \rightarrow N_{S}$ is a $\mathcal{D}_{S}$-precover (respectively, $\mathcal{D}_{S}$-cover) if and only if $\varphi: M_{R} \rightarrow N_{R}$ is a $\mathcal{C}_{R}$-precover (respectively, $\mathcal{C}_{R}$-cover).

(ii) If $\varphi: M_{R} \rightarrow N_{R}$ is a $\mathcal{C}_{R}$-preenvelope (respectively, $\mathcal{C}_{R}$-envelope), then $\varphi: M_{S} \rightarrow N_{S}$ is a $\mathcal{D}_{S}$-preenvelope (respectively, $\mathcal{D}_{S}-$ envelope).

Proof.

(i) follows from Theorem 3.6, Corollary 3.7 and Lemma 4.1.

(ii) holds by Theorem 3.1, Corollary 3.2 and Lemma 4.1.

Following Theorem 4.6, we have

Corollary 4.7. Let $R \rightarrow S$ be a surjective ring homomorphism with $S_{R}$ flat, $\varphi: M_{S} \rightarrow N_{S}$ a right $S$-homomorphism.

(i) $\varphi: M_{S} \rightarrow N_{S}$ is a flat precover (respectively, flat cover) of $S$-modules if and only if $\varphi: M_{R} \rightarrow N_{R}$ is a flat precover (respectively, flat cover) of $R$-modules.

(ii) If $\varphi: M_{R} \rightarrow N_{R}$ is a flat preenvelope (respectively, flat envelope) of $R$-modules, then $\varphi: M_{S} \rightarrow N_{S}$ is a flat preenvelope (respectively, flat envelope) of $S$-modules.

Corollary 4.8. Let $R \rightarrow S$ be a surjective ring homomorphism with $S_{R}$ projective, $\varphi: M_{S} \rightarrow N_{S}$ a right $S$-homomorphism.

(i) $\varphi: M_{S} \rightarrow N_{S}$ is a projective precover (respectively, projective cover) of $S$-modules if and only if $\varphi: M_{R} \rightarrow N_{R}$ is a projective precover (respectively, projective cover) of $R$-modules.

(ii) If $\varphi: M_{R} \rightarrow N_{R}$ is a projective preenvelope (respectively, projective envelope) of $R$-modules, then $\varphi: M_{S} \rightarrow N_{S}$ is a projective preenvelope (respectively, projective envelope) of $S$-modules.

Next, we consider the (pre)covers and (pre)envelopes under (almost) excellent extensions of rings. Recall that a ring $S$ is said to be an almost 
excellent extension of a subring $R$ [14] if the following conditions are satisfied:

(i) $S$ is a finite normalizing extension of $R$, namely, $R$ and $S$ have the same identity and there are elements $s_{1}, \ldots, s_{n} \in S$ such that $S=R s_{1}+\cdots+R s_{n}$ and $R s_{i}=s_{i} R$ for all $i=1, \ldots, n$.

(ii) ${ }_{R} S$ is flat and $S_{R}$ is projective.

(iii) $S$ is right $R$-projective, namely, if $M_{S}$ is a submodule of $N_{S}$ and $M_{R}$ is a direct summand of $N_{R}$, then $M_{S}$ is a direct summand of $N_{S}$.

Further, $S$ is called an excellent extension of $R$ [8] if $S$ is an almost excellent extension of $R$ and $S$ is free with basis $s_{1}, \ldots, s_{n}$ as both a right and a left $R$-module with $s_{1}=1_{R}$.

Theorem 4.9. Let $S$ be an almost excellent extension of a subring $R, \mathcal{C}_{R}$ a class of right $R$-modules, $\mathcal{D}_{S}$ a class of right $S$-modules with $\left(\mathcal{D}_{S}\right)_{R} \subseteq \mathcal{C}_{R}$ and $\left(\operatorname{Hom}_{R}\left(S, \mathcal{C}_{R}\right)\right)_{S} \subseteq \mathcal{D}_{S}, \varphi: M_{R} \rightarrow N_{R}$ a right $R$ homomorphism with $M_{R} \in \mathcal{C}_{R}$. Then the following are equivalent:

(i) $\varphi: M_{R} \rightarrow N_{R}$ is a $\mathcal{C}_{R}$-precover.

(ii) $\varphi_{*}: \operatorname{Hom}_{R}(S, M) \rightarrow \operatorname{Hom}_{R}(S, N)$ is a $\mathcal{D}_{S}$-precover.

(iii) $\varphi_{*}: \operatorname{Hom}_{R}(S, M) \rightarrow \operatorname{Hom}_{R}(S, N)$ is a $\left(\operatorname{Hom}_{R}\left(S, \mathcal{C}_{R}\right)\right)_{S}$-precover.

(iv) $\varphi \varepsilon_{M}: \operatorname{Hom}_{R}(S, M) \rightarrow N_{R}$ is a $\left(\operatorname{Hom}_{R}\left(S, \mathcal{C}_{R}\right)\right)_{R}$-precover.

Proof. By Theorem 2.1, it is enough to show that (iv) $\Rightarrow$ (i).

Let $G_{R} \in \mathcal{C}_{R}$. By [15, Lemma 2.6], there exists a positive integer $t$ such that $G_{R}$ is isomorphic to a direct summand of $\left(\operatorname{Hom}_{R}(S, G)\right)^{t}$.

By (iv), we get the exact sequence

$\operatorname{Hom}_{R}\left(\operatorname{Hom}_{R}(S, G), \operatorname{Hom}_{R}(S, M)\right) \longrightarrow \operatorname{Hom}_{R}\left(\operatorname{Hom}_{R}(S, G), N\right) \longrightarrow 0$, which induces the exact sequence $\operatorname{Hom}_{R}\left(\operatorname{Hom}_{R}(S, G)^{t}, \operatorname{Hom}_{R}(S, M)\right) \longrightarrow \operatorname{Hom}_{R}\left(\operatorname{Hom}_{R}(S, G)^{t}, N\right) \longrightarrow 0$. So we obtain the exact sequence

$$
\operatorname{Hom}_{R}\left(G, \operatorname{Hom}_{R}(S, M) \longrightarrow \operatorname{Hom}_{R}(G, N) \longrightarrow 0,\right.
$$

which gives the exactness of the sequence $\operatorname{Hom}_{R}(G, M) \rightarrow \operatorname{Hom}_{R}(G, N)$ $\rightarrow 0$. Thus, $\varphi: M_{R} \rightarrow N_{R}$ is a $\mathcal{C}_{R}$-precover. 
Theorem 4.10. Let $S$ be an excellent extension of a subring $R$, $\mathcal{C}_{R}$ a class of right $R$-modules, $\mathcal{D}_{S}$ a class of right $S$-modules with $\left(\mathcal{D}_{S}\right)_{R} \subseteq \mathcal{C}_{R}$ and $\left(\operatorname{Hom}_{R}\left(S, \mathcal{C}_{R}\right)\right)_{S} \subseteq \mathcal{D}_{S}, \varphi: M_{S} \rightarrow N_{S}$ a right $S$ homomorphism with $N_{S} \in \mathcal{D}_{S}$. Then the following are equivalent:

(i) $\varphi_{*}: \operatorname{Hom}_{R}(S, M) \rightarrow \operatorname{Hom}_{R}(S, N)$ is a $\mathcal{D}_{S}$-preenvelope.

(ii) $\varphi_{*} \eta_{M}: M_{S} \rightarrow \operatorname{Hom}_{R}(S, N)$ is a $\left(\operatorname{Hom}_{R}\left(S, \mathcal{C}_{R}\right)\right)_{S}$-preenvelope.

(iii) $\varphi: M_{R} \rightarrow N_{R}$ is a $\mathcal{C}_{R}$-preenvelope.

(iv) $\varphi: M_{S} \rightarrow N_{S}$ is a $\mathcal{D}_{S}$-preenvelope.

Proof. (i) $\Rightarrow$ (ii) $\Leftrightarrow$ (iii) follow from Theorem 2.3.

(iii) $\Rightarrow$ (i). For any $A_{S} \in \mathcal{D}_{S}, A_{R} \in \mathcal{C}_{R}$. Thus, we get the exact sequence $\operatorname{Hom}_{R}(N, A) \rightarrow \operatorname{Hom}_{R}(M, A) \rightarrow 0$. Since $S$ is an excellent

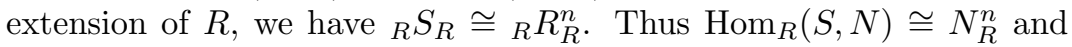
$\operatorname{Hom}_{R}(S, M) \cong M_{R}^{n}$. So we get the exact sequence

$\operatorname{Hom}_{R}\left(\operatorname{Hom}_{R}(S, N) \otimes_{S} S, A\right) \longrightarrow \operatorname{Hom}_{R}\left(\operatorname{Hom}_{R}(S, M) \otimes_{S} S, A\right) \longrightarrow 0$, which induces the exact sequence

$\operatorname{Hom}_{S}\left(\operatorname{Hom}_{R}(S, N), \operatorname{Hom}_{R}(S, A)\right)$ $\longrightarrow \operatorname{Hom}_{S}\left(\operatorname{Hom}_{R}(S, M), \operatorname{Hom}_{R}(S, A)\right) \longrightarrow 0$.

Since $A_{S}$ is isomorphic to a direct summand of $\operatorname{Hom}_{R}(S, A)$ by [14, Lemma 1.1], we have the exact sequence

$\operatorname{Hom}_{S}\left(\operatorname{Hom}_{R}(S, N), A_{S}\right) \longrightarrow \operatorname{Hom}_{S}\left(\operatorname{Hom}_{R}(S, M), A_{S}\right) \longrightarrow 0$.

So $\varphi_{*}: \operatorname{Hom}_{R}(S, M) \rightarrow \operatorname{Hom}_{R}(S, N)$ is a $\mathcal{D}_{S}$-preenvelope.

(i) $\Rightarrow$ (iv) $\Rightarrow$ (ii) are straightforward by [14, Lemma 1.1].

Theorem 4.11. Let $S$ be an excellent extension of a subring $R$, $\mathcal{C}_{R}$ a class of right $R$-modules, $\mathcal{D}_{S}$ a class of right $S$-modules with $\left(\mathcal{D}_{S}\right)_{R} \subseteq \mathcal{C}_{R}$ and $\left(\mathcal{C}_{R} \otimes_{R} S\right)_{S} \subseteq \mathcal{D}_{S}, \varphi: M_{S} \rightarrow N_{S}$ a right $S$ homomorphism with $M_{S} \in \mathcal{D}_{S}$. Then the following are equivalent:

(i) $\varphi \otimes_{R} 1: M \otimes_{R} S \rightarrow N \otimes_{R} S$ is a $\mathcal{D}_{S}$-precover.

(ii) $\mu_{N}\left(\varphi \otimes_{R} 1\right): M \otimes_{R} S \rightarrow N_{S}$ is a $\left(\mathcal{C}_{R} \otimes_{R} S\right)_{S}$-precover.

(iii) $\varphi: M_{R} \rightarrow N_{R}$ is a $\mathcal{C}_{R}$-precover.

(iv) $\varphi: M_{S} \rightarrow N_{S}$ is a $\mathcal{D}_{S}$-precover. 
Proof. (i) $\Rightarrow$ (iv) is easy by $[\mathbf{1 4}$, Lemma 1.1]. (iv) $\Rightarrow$ (ii) $\Leftrightarrow$ (iii) hold by Theorem 3.6.

(iii) $\Rightarrow$ (i). For any $A_{S} \in \mathcal{D}_{S}, A_{R} \in \mathcal{C}_{R}$. Therefore, we get the exact sequence $\operatorname{Hom}_{R}(A, M) \rightarrow \operatorname{Hom}_{R}(A, N) \rightarrow 0$. Since ${ }_{R} S_{R} \cong{ }_{R} R_{R}^{n}$, we have $M \otimes_{R} S \cong M_{R}^{n}$ and $N \otimes_{R} S \cong N_{R}^{n}$. So we get the exact sequence

$\operatorname{Hom}_{R}\left(A, \operatorname{Hom}_{S}\left(S, M \otimes_{R} S\right)\right) \rightarrow \operatorname{Hom}_{R}\left(A, \operatorname{Hom}_{S}\left(S, N \otimes_{R} S\right)\right) \rightarrow 0$,

which induces the exact sequence

$$
\operatorname{Hom}_{S}\left(A \otimes_{R} S, M \otimes_{R} S\right) \longrightarrow \operatorname{Hom}_{S}\left(A \otimes_{R} S, N \otimes_{R} S\right) \longrightarrow 0 .
$$

Since $A_{S}$ is isomorphic to a direct summand of $A \otimes_{R} S$, by [14, Lemma 1.1], we have the exact sequence $\operatorname{Hom}_{S}\left(A_{S}, M \otimes_{R} S\right) \rightarrow$ $\operatorname{Hom}_{S}\left(A_{S}, N \otimes_{R} S\right) \rightarrow 0$. Thus, $\varphi \otimes_{R} 1: M \otimes_{R} S \rightarrow N \otimes_{R} S$ is a $\mathcal{D}_{S}$-precover.

Theorem 4.12. Let $S$ be an excellent extension of a subring $R$, $\mathcal{C}_{R}$ a class of right $R$-modules, $\mathcal{D}_{S}$ a class of right $S$-modules with $\left(\mathcal{D}_{S}\right)_{R} \subseteq \mathcal{C}_{R}$ and $\left(\mathcal{C}_{R} \otimes_{R} S\right)_{S} \subseteq \mathcal{D}_{S}, \varphi: M_{R} \rightarrow N_{R}$ a right $R$ homomorphism with $N_{R} \in \mathcal{C}_{R}$. Then the following are equivalent:

(i) $\varphi: M_{R} \rightarrow N_{R}$ is a $\mathcal{C}_{R}$-preenvelope.

(ii) $\varphi \otimes_{R} 1: M \otimes_{R} S \rightarrow N \otimes_{R} S$ is a $\mathcal{D}_{S}$-preenvelope.

(iii) $\varphi \otimes_{R} 1: M \otimes_{R} S \rightarrow N \otimes_{R} S$ is a $\left(\mathcal{C}_{R} \otimes_{R} S\right)_{S}$-preenvelope.

(iv) $\nu_{N} \varphi: M_{R} \rightarrow N \otimes_{R} S$ is a $\left(\mathcal{C}_{R} \otimes_{R} S\right)_{R}$-preenvelope.

Proof. By Theorem 3.1, it is enough to show that (iv) $\Rightarrow$ (i).

Let $G_{R} \in \mathcal{C}_{R}$. Since ${ }_{R} S_{R} \cong{ }_{R} R_{R}^{n}, G \otimes_{R} S \cong G_{R}^{n}$. By (iv), we get the exact sequence

$$
\operatorname{Hom}_{R}\left(N \otimes_{R} S, G \otimes_{R} S\right) \longrightarrow \operatorname{Hom}_{R}\left(M, G \otimes_{R} S\right) \longrightarrow 0,
$$

which induces the exact sequence

$$
\operatorname{Hom}_{R}\left(N \otimes_{R} S, G\right)^{n} \longrightarrow \operatorname{Hom}_{R}(M, G)^{n} \longrightarrow 0 .
$$

Thus, we obtain the exact sequence

$$
\operatorname{Hom}_{R}\left(N \otimes_{R} S, G\right) \longrightarrow \operatorname{Hom}_{R}(M, G) \rightarrow 0,
$$

which yields the exactness of the sequence $\operatorname{Hom}_{R}(N, G) \rightarrow \operatorname{Hom}_{R}(M, G)$ $\rightarrow 0$. So $\varphi: M_{R} \rightarrow N_{R}$ is a $\mathcal{C}_{R}$-preenvelope. 
Finally, we investigate the special preenvelopes and precovers under (almost) excellent extensions.

Theorem 4.13. Let $S$ be an almost excellent extension of a subring $R, \mathcal{C}_{R}$ a class of right $R$-modules, $\mathcal{D}_{S}$ a class of right $S$-modules with $\left(\mathcal{D}_{S}\right)_{R} \subseteq \mathcal{C}_{R}$ and $\left(\operatorname{Hom}_{R}\left(S, \mathcal{C}_{R}\right)\right)_{S} \subseteq \mathcal{D}_{S}, \varphi: M_{S} \rightarrow N_{S}$ a right $S$ homomorphism with $N_{S} \in \mathcal{D}_{S}$. Consider the following conditions:

(i) $\varphi_{*}: \operatorname{Hom}_{R}(S, M) \rightarrow \operatorname{Hom}_{R}(S, N)$ is a special $\mathcal{D}_{S}$-preenvelope.

(ii) $\varphi: M_{S} \rightarrow N_{S}$ is a special $\mathcal{D}_{S}$-preenvelope.

(iii) $\varphi: M_{R} \rightarrow N_{R}$ is a special $\mathcal{C}_{R}$-preenvelope.

Then (i) $\Rightarrow$ (ii) $\Leftrightarrow$ (iii). Moreover, if $S$ is an excellent extension of $R$, then (ii) $\Rightarrow$ (i).

Proof.

(i) $\Rightarrow$ (ii). Since $\varphi_{*}: \operatorname{Hom}_{R}(S, M) \rightarrow \operatorname{Hom}_{R}(S, N)$ is monic, $\varphi$ is monic by [14, Lemma 1.1]. So there is an exact sequence $0 \rightarrow M_{S} \rightarrow N_{S} \rightarrow L_{S} \rightarrow 0$ of right $S$-modules. Applying the functor $\operatorname{Hom}_{R}(S,-)$ to it, we get the exact sequence

$$
0 \rightarrow \operatorname{Hom}_{R}(S, M) \longrightarrow \operatorname{Hom}_{R}(S, N) \longrightarrow \operatorname{Hom}_{R}(S, L) \longrightarrow 0
$$

of right $S$-modules with $\operatorname{Hom}_{R}(S, L) \in{ }^{\perp} \mathcal{D}_{S}$. Since $L_{S}$ is isomorphic to a direct summand of $\operatorname{Hom}_{R}(S, L)$. $L_{S} \in{ }^{\perp} \mathcal{D}_{S}$. So $\varphi: M_{S} \rightarrow N_{S}$ is a special $\mathcal{D}_{S}$-preenvelope.

(ii) $\Rightarrow$ (iii) follows from Proposition 2.8 (ii).

(iii) $\Rightarrow$ (ii). By (iii), there is an exact sequence $0 \rightarrow M_{S} \rightarrow$ $N_{S} \rightarrow L_{S} \rightarrow 0$ of right $S$-modules with $L_{R} \in{ }^{\perp} \mathcal{C}_{R}$. For any $B_{S} \in \mathcal{D}_{S}, B_{R} \in \mathcal{C}_{R}$. Thus, by [10, Theorem 11.66], we have $\operatorname{Ext}_{S}^{1}\left(L, \operatorname{Hom}_{R}(S, B)\right) \cong \operatorname{Ext}_{R}^{1}(L, B)=0$. But, $B_{S}$ is isomorphic to a direct summand of $\operatorname{Hom}_{R}(S, B)$. Hence, $\operatorname{Ext}_{S}^{1}(L, B)=0$, and so $L_{S} \in{ }^{\perp} \mathcal{D}_{S}$. Thus $\varphi: M_{S} \rightarrow N_{S}$ is a special $\mathcal{D}_{S}$-preenvelope.

(ii) $\Rightarrow$ (i). There is an exact sequence $0 \rightarrow M_{S} \rightarrow N_{S} \rightarrow$ $L_{S} \rightarrow 0$ of right $S$-modules with $L_{S} \in{ }^{\perp} \mathcal{D}_{S}$, which induces the right $S$-module exact sequence $0 \rightarrow \operatorname{Hom}_{R}(S, M) \rightarrow \operatorname{Hom}_{R}(S, N) \rightarrow$ $\operatorname{Hom}_{R}(S, L) \rightarrow 0$. Since ${ }_{R} S_{R} \cong{ }_{R} R_{R}^{n}, \operatorname{Hom}_{R}(S, L) \cong L_{R}^{n}$. Let $A_{S} \in$ $\mathcal{D}_{S}$. Then $\operatorname{Hom}_{R}(S, A) \in \mathcal{D}_{S}$. $\operatorname{So}_{\operatorname{Ext}_{S}^{1}}\left(\operatorname{Hom}_{R}(S, L), \operatorname{Hom}_{R}(S, A)\right) \cong$ $\operatorname{Ext}_{R}^{1}\left(L^{n}, A\right) \cong \operatorname{Ext}_{R}^{1}(L, A)^{n} \cong \operatorname{Ext}_{S}^{1}\left(L, \operatorname{Hom}_{R}(S, A)\right)^{n}=0$. Since $A_{S}$ 
is isomorphic to a direct summand of $\operatorname{Hom}_{R}(S, A), \operatorname{Hom}_{R}(S, L) \in{ }^{\perp} \mathcal{D}_{S}$. It follows that $\varphi_{*}: \operatorname{Hom}_{R}(S, M) \rightarrow \operatorname{Hom}_{R}(S, N)$ is a special $\mathcal{D}_{S^{-}}$ preenvelope since $\operatorname{Hom}_{R}(S, N) \in \mathcal{D}_{S}$.

Theorem 4.14. Let $S$ be an almost excellent extension of a subring $R, \mathcal{C}_{R}$ a class of right $R$-modules, $\mathcal{D}_{S}$ a class of right $S$-modules with $\left(\mathcal{D}_{S}\right)_{R} \subseteq \mathcal{C}_{R}$ and $\left(\operatorname{Hom}_{R}\left(S, \mathcal{C}_{R}\right)\right)_{S} \subseteq \mathcal{D}_{S}, \varphi: M_{S} \rightarrow N_{S}$ a right $S$ homomorphism with $M_{S} \in \mathcal{D}_{S}$. Consider the following conditions:

(i) $\varphi: M_{R} \rightarrow N_{R}$ is a special $\mathcal{C}_{R}$-precover.

(ii) $\varphi_{*}: \operatorname{Hom}_{R}(S, M) \rightarrow \operatorname{Hom}_{R}(S, N)$ is a special $\mathcal{D}_{S}$-precover.

(iii) $\varphi: M_{S} \rightarrow N_{S}$ is a special $\mathcal{D}_{S}$-precover.

Then (i) $\Rightarrow$ (ii) $\Rightarrow$ (iii). Moreover, if $S$ is an excellent extension of $R$, then (ii) $\Rightarrow$ (i).

Proof. (i) $\Rightarrow$ (ii) follows from Proposition 2.8 (i).

(ii) $\Rightarrow$ (iii). Since $\varphi_{*}: \operatorname{Hom}_{R}(S, M) \rightarrow \operatorname{Hom}_{R}(S, N)$ is epic, $\varphi$ is epic by [14, Lemma 1.1]. So there is an exact sequence $0 \rightarrow K_{S} \rightarrow$ $M_{S} \rightarrow N_{S} \rightarrow 0$ of right $S$-modules, which induces the exact sequence $0 \rightarrow \operatorname{Hom}_{R}(S, K) \rightarrow \operatorname{Hom}_{R}(S, M) \rightarrow \operatorname{Hom}_{R}(S, N) \rightarrow 0$ of right $S$ modules with $\operatorname{Hom}_{R}(S, K) \in \mathcal{D}_{S}^{\perp}$. Since $K_{S}$ is isomorphic to a direct summand of $\operatorname{Hom}_{R}(S, K), K_{S} \in \mathcal{D} \frac{\perp}{S}$. So $\varphi: M_{S} \rightarrow N_{S}$ is a special $\mathcal{D}_{S}$-precover.

(ii) $\Rightarrow$ (i). By (ii), there is an exact sequence $0 \rightarrow K_{R} \rightarrow M_{R} \rightarrow$ $N_{R} \rightarrow 0$ of right $R$-modules. For any $A_{R} \in \mathcal{C}_{R}, \operatorname{Hom}_{R}(S, A) \in \mathcal{D}_{S}$. Also, ${ }_{R} S_{R} \cong{ }_{R} R_{R}^{n}, \operatorname{Hom}_{R}(S, A) \cong A_{R}^{n}$. Hence, $\operatorname{Ext}_{R}^{1}\left(A^{n}, K\right) \cong$ $\operatorname{Ext}_{R}^{1}\left(\operatorname{Hom}_{R}(S, A), K\right) \cong \operatorname{Ext}_{S}^{1}\left(\operatorname{Hom}_{R}(S, A), \operatorname{Hom}_{R}(S, K)\right)=0$. So $\operatorname{Ext}_{R}^{1}(A, K)=0$. Thus $\varphi: M_{R} \rightarrow N_{R}$ is a special $\mathcal{C}_{R}$-precover.

Theorem 4.15. Let $S$ be an almost excellent extension of a subring $R, \mathcal{C}_{R}$ a class of right $R$-modules, $\mathcal{D}_{S}$ a class of right $S$-modules with $\left(\mathcal{D}_{S}\right)_{R} \subseteq \mathcal{C}_{R}$ and $\left(\mathcal{C}_{R} \otimes_{R} S\right)_{S} \subseteq \mathcal{D}_{S}, \varphi: M_{S} \rightarrow N_{S}$ a right $S$ homomorphism with $M_{S} \in \mathcal{D}_{S}$. Consider the following conditions:

(i) $\varphi \otimes_{R} 1: M \otimes_{R} S \rightarrow N \otimes_{R} S$ is a special $\mathcal{D}_{S}$-precover.

(ii) $\varphi: M_{S} \rightarrow N_{S}$ is a special $\mathcal{D}_{S}$-precover.

(iii) $\varphi: M_{R} \rightarrow N_{R}$ is a special $\mathcal{C}_{R}$-precover. 
Then (i) $\Rightarrow$ (ii) $\Leftrightarrow$ (iii). Moreover, if $S$ is an excellent extension of $R$, then (ii) $\Rightarrow$ (i).

\section{Proof.}

(i) $\Rightarrow$ (ii). Since $\varphi \otimes_{R} 1: M \otimes_{R} S \rightarrow N \otimes_{R} S$ is epic, $\varphi$ is epic by [14, Lemma 1.1]. So there is an exact sequence $0 \rightarrow K_{S} \rightarrow M_{S} \rightarrow N_{S} \rightarrow 0$ of right $S$-modules. Applying the functor $-\otimes_{R} S$ to it, we get the exact sequence $0 \rightarrow K \otimes_{R} S \rightarrow M \otimes_{R} S \rightarrow N \otimes_{R} S \rightarrow 0$ of right $S$-modules with $K \otimes_{R} S \in \mathcal{D} \perp$. Since $K_{S}$ is isomorphic to a direct summand of $K \otimes_{R} S, K_{S} \in \mathcal{D} \frac{\perp}{S}$. So $\varphi: M_{S} \rightarrow N_{S}$ is a special $\mathcal{D}_{S}$-precover.

(ii) $\Rightarrow$ (iii) follows from Proposition 3.9 (i).

(iii) $\Rightarrow$ (ii). There is an exact sequence $0 \rightarrow K_{S} \rightarrow M_{S} \rightarrow N_{S} \rightarrow 0$ of right $S$-modules with $K_{R} \in \mathcal{C}_{R}^{\perp}$. For any $A_{S} \in \mathcal{D}_{S}, A_{R} \in \mathcal{C}_{R}$. So $\operatorname{Ext}_{S}^{1}\left(A \otimes_{R} S, K\right) \cong \operatorname{Ext}_{R}^{1}(A, K)=0$ by [10, Theorem 11.65]. But $A_{S}$ is isomorphic to a direct summand of $A \otimes_{R} S$, and so $\operatorname{Ext}_{S}^{1}\left(A_{S}, K_{S}\right)=0$. Thus, $K_{S} \in \mathcal{D} \frac{\perp}{S}$. Hence, $\varphi: M_{S} \rightarrow N_{S}$ is a special $\mathcal{D}_{S}$-precover.

(ii) $\Rightarrow$ (i). There is an exact sequence $0 \rightarrow K_{S} \rightarrow M_{S} \rightarrow N_{S} \rightarrow 0$ of right $S$-modules with $K_{S} \in \mathcal{D}_{S}^{\perp}$, which induces the exact sequence $0 \rightarrow K \otimes_{R} S \rightarrow M \otimes_{R} S \rightarrow N \otimes_{R} S \rightarrow 0$ of right $S$-modules. Since $S$ is an excellent extension of $R,{ }_{R} S_{R} \cong{ }_{R} R_{R}^{n}$. Thus, $K \otimes_{R} S \cong K_{R}^{n}$. For any $A_{S} \in \mathcal{D}_{S}, A \otimes_{R} S \in \mathcal{D}_{S}$, and so we have

$$
\begin{aligned}
\operatorname{Ext}_{S}^{1}\left(A \otimes_{R} S, K \otimes_{R} S\right) & \cong \operatorname{Ext}_{R}^{1}\left(A, K^{n}\right) \cong \operatorname{Ext}_{R}^{1}(A, K)^{n} \\
& \cong \operatorname{Ext}_{S}^{1}\left(A \otimes_{R} S, K\right)^{n}=0 .
\end{aligned}
$$

But $A_{S}$ is isomorphic to a direct summand of $A \otimes_{R} S$. So $K \otimes_{R} S \in \mathcal{D} \frac{\perp}{\perp}$, whence $\varphi \otimes_{R} 1: M \otimes_{R} S \rightarrow N \otimes_{R} S$ is a special $\mathcal{D}_{S}$-precover.

Theorem 4.16. Let $S$ be an almost excellent extension of a subring $R, \mathcal{C}_{R}$ a class of right $R$-modules, $\mathcal{D}_{S}$ a class of right $S$-modules with $\left(\mathcal{D}_{S}\right)_{R} \subseteq \mathcal{C}_{R}$ and $\left(\mathcal{C}_{R} \otimes_{R} S\right)_{S} \subseteq \mathcal{D}_{S}, \varphi: M_{S} \rightarrow N_{S}$ a right $S$ homomorphism with $N_{S} \in \mathcal{D}_{S}$. Consider the following conditions:

(i) $\varphi: M_{R} \rightarrow N_{R}$ is a special $\mathcal{C}_{R}$-preenvelope.

(ii) $\varphi \otimes_{R} 1: M \otimes_{R} S \rightarrow N \otimes_{R} S$ is a special $\mathcal{D}_{S}$-preenvelope.

(iii) $\varphi: M_{S} \rightarrow N_{S}$ is a special $\mathcal{D}_{S}$-preenvelope.

Then (i) $\Rightarrow$ (ii) $\Rightarrow$ (iii). Moreover, if $S$ is an excellent extension of $R$, then (ii) $\Rightarrow$ (i). 
Proof.

(i) $\Rightarrow$ (ii) follows from Proposition 3.9 (ii).

(ii) $\Rightarrow$ (iii). Since $\varphi \otimes_{R} 1: M \otimes_{R} S \rightarrow N \otimes_{R} S$ is monic, $\varphi$ is monic by [14, Lemma 1.1]. So there is an exact sequence $0 \rightarrow M_{S} \rightarrow$ $N_{S} \rightarrow L_{S} \rightarrow 0$ of right $S$-modules, which induces the exact sequence $0 \rightarrow M \otimes_{R} S \rightarrow N \otimes_{R} S \rightarrow L \otimes_{R} S \rightarrow 0$ of right $S$-modules with $L \otimes_{R} S \in{ }^{\perp} \mathcal{D}_{S}$. Since $L_{S}$ is isomorphic to a direct summand of $L \otimes_{R} S$, $L_{S} \in{ }^{\perp} \mathcal{D}_{S}$. So $\varphi: M_{S} \rightarrow N_{S}$ is a special $\mathcal{D}_{S}$-preenvelope.

(ii) $\Rightarrow$ (i). There is an exact sequence $0 \rightarrow M_{R} \rightarrow N_{R} \rightarrow L_{R} \rightarrow 0$ of right $R$-modules, which induces the exact sequence $0 \rightarrow M \otimes_{R} S \rightarrow$ $N \otimes_{R} S \rightarrow L \otimes_{R} S \rightarrow 0$ of right $S$-modules with $L \otimes_{R} S \in{ }^{\perp} \mathcal{D}_{S}$. For any $A_{R} \in \mathcal{C}_{R}, A \otimes_{R} S \in \mathcal{D}_{S}$. Since ${ }_{R} S_{R} \cong{ }_{R} R_{R}^{n}, A \otimes_{R} S \cong A_{R}^{n}$. Hence, $\operatorname{Ext}_{R}^{1}\left(L, A^{n}\right) \cong \operatorname{Ext}_{R}^{1}\left(L, A \otimes_{R} S\right) \cong \operatorname{Ext}_{S}^{1}\left(L \otimes_{R} S, A \otimes_{R} S\right)=0$. So $\operatorname{Ext}_{R}^{1}(L, A)=0$. Thus, $\varphi: M_{R} \rightarrow N_{R}$ is a special $\mathcal{C}_{R}$-preenvelope.

Acknowledgments. The author wishes to express his gratitude to the referee for his/her helpful suggestions and comments for the improvement of the paper.

\section{REFERENCES}

1. F.W. Anderson and K.R. Fuller, Rings and categories of modules, SpringerVerlag, New York, 1974.

2. M. Auslander and S. Smalø, Preprojective modules over artin algrbras, J. Algebra 66 (1980), 61-122.

3. D. Dempsey, L. Oyonarte and Y.M. Song, Ring extensions, injective covers and envelopes, Arch. Math. 76 (2001), 250-258.

4. E.E. Enochs, Injective and flat covers, envelopes and resolvents, Israel J. Math. 39 (1981), 189-209.

5. E.E. Enochs and O.M.G. Jenda, Relative homological algebra, Walter de Gruyter, Berlin, 2000.

6. R. Göbel and J. Trlifaj, Approximations and endomorphism algebras of modules, GEM 41, Walter de Gruyter, Berlin, 2006.

7. T.Y. Lam, Lectures on modules and rings, Springer-Verlag, New York, 1999.

8. D.S. Passman, The algebraic structure of group rings, Wiley-Interscience, New York, 1977.

9. K.R. Pinzon, Absolutely pure modules, University of Kentucky, Doctoral thesis, 2005.

10. J.J. Rotman, An introduction to homological algebra, Academic Press, New York, 1979. 
11. B. Stenström, Coherent rings and FP-injective modules, J. Lond. Math. Soc. 2 (1970), 323-329.

12. T. Würfel, Ring extensions and essential monomorphisms, Proc. Amer. Math. Soc. 69 (1978), 1-7.

13. J. Xu, Flat covers of modules, Lect. Notes Math. 1634, Springer-Verlag, Berlin, 1996.

14. W.M. Xue, On almost excellent extensions, Alg. Colloq. 3 (1996), 125-134.

15. D.X. Zhou, Cotorsion pair extensions, Acta Math. Sin. 25 (2009), 15671582 .

Department of Mathematics and Physics, Nanjing Institute of Technology, NANJing 211167, China

Email address: maolx2@hotmail.com 